\title{
The Search for Putative Hits in Combating Leishmaniasis: The Contributions of Natural Products Over the Last Decade
}

\author{
Patrick O. Sakyi ${ }^{1,2} \cdot$ Richard K. Amewu $^{1} \cdot$ Robert N. O. A. Devine ${ }^{2} \cdot$ Emahi Ismaila $^{2} \cdot$ Whelton A. Miller ${ }^{3,4,5}$. \\ Samuel K. Kwofie ${ }^{6,7}$ (])
}

Received: 11 February 2021 / Accepted: 7 May 2021 / Published online: 14 July 2021

(c) The Author(s) 2021

\begin{abstract}
Despite advancements in the areas of omics and chemoinformatics, potent novel biotherapeutic molecules with new modes of actions are needed for leishmaniasis. The socioeconomic burden of leishmaniasis remains alarming in endemic regions. Currently, reports from existing endemic areas such as Nepal, Iran, Brazil, India, Sudan and Afghanistan, as well as newly affected countries such as Peru, Bolivia and Somalia indicate concerns of chemoresistance to the classical antimonial treatment. As a result, effective antileishmanial agents which are safe and affordable are urgently needed. Natural products from both flora and fauna have contributed immensely to chemotherapeutics and serve as vital sources of new chemical agents. This review focuses on a systematic cross-sectional view of all characterized anti-leishmanial compounds from natural sources over the last decade. Furthermore, $\mathrm{IC}_{50} / \mathrm{EC}_{50}$, cytotoxicity and suggested mechanisms of action of some of these natural products are provided. The natural product classification includes alkaloids, terpenes, terpenoids, and phenolics. The plethora of reported mechanisms involve calcium channel inhibition, immunomodulation and apoptosis. Making available enriched data pertaining to bioactivity and mechanisms of natural products complement current efforts geared towards unraveling potent leishmanicides of therapeutic relevance.
\end{abstract}

\section{Graphic Abstract}

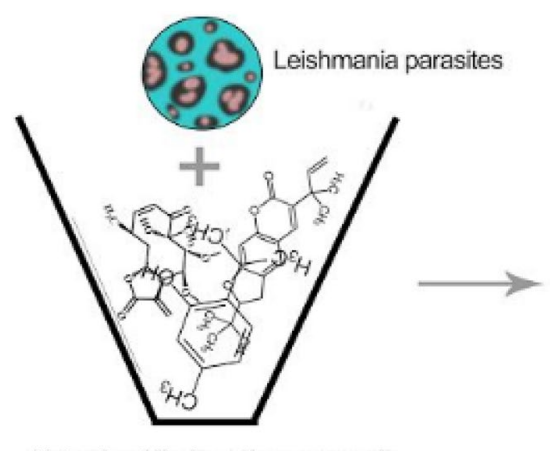

Natural anti-lieshmania compounds

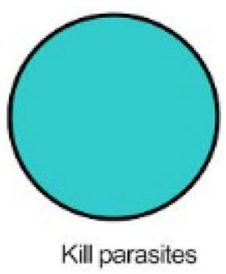

Keywords Chemotherapeutics $\cdot$ Chemoinformatics $\cdot$ Natural products $\cdot$ Cytotoxicity $\cdot$ Leishmaniasis $\cdot$ Phenotypic screening

Samuel K. Kwofie

skkwofie@ug.edu.gh

Extended author information available on the last page of the article

\section{Introduction}

The debilitating rate of parasitic infections in the tropical and subtropical regions of developing countries has become alarming [1]. Vector-borne neglected tropical diseases and related synergetic co-infections, particularly leishmaniasis are very challenging and sophisticated to treat [2]. This 
is partly due to the existence of diverse parasitic species with different bionomics and sophisticated overlap between virulent factors. Activated immune response during disease exacerbation coupled with emerging resistance by both parasites and vectors against various treatment regimens have also contributed to this challenge [2,3].

Leishmania, the etiological agent of leishmaniasis, is transmitted globally by over 90 different female sand-fly species of the Phlebotomus family, spread across 98 countries and four continents, with annual estimates of 1 million new cases and 30,000 deaths as at 2017 [2,4]. The exact disease burden is unknown, but statistics indicate that over 350 million people are at risk, signifying a prominent public health risk $[2,5,6]$.

Leishmaniasis is curable if the disease is diagnosed early and the appropriate medication is administered. Typically, leishmaniasis is initially marked by dermotropic ulcers, which then progress into the visceral tissues, resulting in a late and more debilitating condition that can often lead to death if left untreated. In some cases, the destruction of the mucocutaneous membrane especially the nose, throat, and mouth have also been very common [2]. The degree of clinical outcome and its corresponding immunopathology depends primarily on the type of causative species, age of host, and the balance between the host immune response and how the parasites subvert these defense mechanisms. In cases where the victim's immune system is strong, Leishmania pathogens behave as opportunists by remaining dormant until the host's immunity is compromised. Additionally, when the host is immunosuppressed, relapses are usually prevalent resulting in treatment failures.

Some challenges associated with the management of leishmaniasis include systemic toxicity of administrated drugs, high cost of existing therapeutic options, lengthy treatment periods and drug resistance. Furthermore, confounding factors such as parasite diversity has hampered various intervention strategies and halted global efforts, necessitating an immediate search for new drug leads for development as the next generation of antileishmanial agents [7-9]. In lieu of this, the review seeks to bring to the fore the various classes of natural products recently discovered with antileishmanial potentials over the last decade. Even though, the review primarily reported compounds with potent bioactivity, few with low potency were reported since these could be optimized or their scaffolds may serve as skeletons for the development of future leishmanicides.

\subsection{Trends in leishmanial chemotherapy and current panorama}

Protection against leishmaniasis started with mimicking natural immunity through live inoculations [10] until modernized techniques including killed promastigotes and knocked out parasites came into play. Unfortunately, the presence of persistent lesions and the difficulty in estimating their efficacy rendered these approaches less effective [10,11]. Efforts to alleviate leishmaniasis via chemotherapy include the use of pentavalent antimonial, which was essentially a small tartrate complex of antimony first reported in 1925 by Brahmachari $[12,13]$. Although, antimoniate $\left(\mathrm{Sb}^{\mathrm{V}}\right)$ is still active after reduction by arsenate reductase to $\mathrm{Sb}^{\mathrm{III}}$, Leishmania parasites are also susceptible to $\mathrm{Sb}^{\mathrm{V}}$ via oxidative stress.

Gene amplification studies involving the Adenosine Triphosphate (ATP) binding cassette transporters including the multi-resistance proteins that act as efflux pumps have been shown to contribute to antimony resistance in clinical isolates $[14,15]$. Likewise, deletions of aquaporin membrane carrier genes and phenotypic changes of the parasite with subsequent induced effects on the microbicide activity and the efflux rate of antimony reaching the macrophages also contribute to the resistance [16].

In the mid-1960's pentamidine became the second choice to antimony resistant strains [17]. However, its utility like the antimonial was hampered due to severe vasomotor side effects and complex interactions with the pancreas which leads to the destruction of $\beta$-cells causing diabetes mellitus [17].

In the quest to expedite the time it takes for drugs to reach the market, strategies such as deciphering the cellular similarities between disease causing pathogens from phenotypic screening were developed. In the early 1960s, the anti-fungal amphotericin B from Streptomyces nodosus was used for treating leishmaniasis $[18,19]$. This choice was widely accepted in most endemic areas due to its efficacy but not so in other areas especially East Africa (L. donovani) and South America (L. infantum) [20].

The anticancer agent alkyl phosphocholine (miltefosine) was the first oral formulation with strong protection against visceral leishmaniasis. Miltefosine works by modulating an apoptosis process induced by mitochondria membrane depolarization and phospholipid biosynthesis inhibition [21]. The main drawback in administering miltefosine for leishmaniasis treatment includes longer elimination time, lengthy treatment course, and miscarriage in pregnant patients after use [22].

A new and simple formulation of an old antibiotic paromomycin which inhibited translation with different modes of application (enteral, parenteral and topical) was also repurposed for leishmaniasis in 1967 [23, 24]. Unlike the other treatment options, paromomycin's toxic effects are very minimal, but its efficacy is quite poor. New optimum carriers targeting pathogen macrophage using albumin has recently been reported to increase efficiency [25].

Following the failure of miltefosine, a collaboration between the Walter Reed Army Institute of Research 
(WRAIR, USA) and GlaxoSmithKline (UK) identified sitamaquine as a promising alternative, but its apparent loss of efficacy in tegumentary leishmaniasis limited its use [26]. Subsequently, findings from amphotericin B use and its high curative rate in patients influenced another repurposing strategy using the oral anti-fungal azoles (fluconazole, itraconazole, and ketoconazole) as suitable control and cost-effective therapy $[27,28]$.

Due to the therapeutic challenges, new chemotypes with high potency in tandem with immunostimulatory activity targeting new proteins applicable to both visceral and cutaneous leishmaniasis cases are desperately needed.

\subsection{Natural products as possible sources of new drugs against leishmaniasis}

The lack of effective vaccines for control and concerted elimination campaign [2], and recent snail paced progress on leishmanial vaccine development does not guarantee any optimism. With the advancements in synthetic organic chemistry, combinatorial chemistry, and computational de novo drug discovery strategies, as well as high throughput screening techniques, only a few synthetically constructed drugs have been useful in combating leishmaniasis. Even with this, few natural product scaffolds represent major pharmacophores responsible for their curative effects. Between 2005 and 2010, about 19 natural products were registered for treatment of infectious diseases [29]. Similarly, over 69\% of new small molecules used for the treatment of infectious diseases originated from natural products [30,31].

Despite the large molecular weights of natural products which renders some of them less druglike, structural diversity, large chemical space and safety are characteristics that overrides synthetic alternatives. Treatments using extracts from plant families from endemic regions include Fabaceae [32], Annonaceae, Euphorbiaceae [31, 33, 34], Rutaceae [35-37], Myrsinaceae [31, 38], Liliaceae [39], Araliaceae [38], Simaroubaceae [40], as well as endophytes genera Alternaria [41], Arthrinium, Penicillium, Cochloibus, Fusarium, Colletotrichum, and Gibberella [42]. Additionally, the exploration of marine natural products has led to the identification of interesting natural products with diverse biomolecular functions [43, 44].

Since the mid-eighties when the search for anti-leishmanial natural products became prominent, numerous metabolites originating from plants to current antileishmanial therapies have been reported. Lately, credible chemical entities from marine sources and endophytic species have also been reviewed [45-51]. This review presents the various classes of natural products from both flora and fauna that have been isolated over the last decade with anti-leishmanial properties. Also, the $\mathrm{IC}_{50} / \mathrm{EC}_{50}$ values and suggested mechanisms of action of these natural products are discussed.

\subsection{Classification of natural products with anti-leishmania properties}

\subsubsection{Alkaloids}

Among the characterized bioactive constituents from nature, alkaloids have provided a broad-spectrum activity against different ailments and demonstrated their suitability as potential drug leads. Phenotypic alterations in ultrastructure form of the infective cells and immunomodulatory investigation studies of isolated alkaloids within the last decade reveal 27 alkaloids (Table 1) with varying efficacies from strong to weak activity. The natural product 3 isolated from Cissampelos sympodialis acts as a calcium channel inhibitor with immunomodulatory effects through the enhancement of nitric oxide (NO) production in macrophages [52]. Studies of $\mathbf{4}$ from Croton pullei reported significant alterations in organelle membranes of the endoplasmic reticulum, kinoplast and golgi body, depicting an apoptosis-like process [53]. Treatment with spectaline alkaloids, $\mathbf{1 6}$ and $\mathbf{1 7}$ from dichloromethane fractions of the flower Senna spectabilis of Leishmania promastigotes also portray a similar molecular mechanism like its structurally related piperine amide alkaloid, which either modulates the sterol biosynthetic pathway or acts as an inhibitor of cell proliferation by mitochondrion organelle destruction [54]. Although, the exact mode of action has not been fully elucidated, 21 from Berberine vulgaris like the active alkaloid in Berberine aristate perpetuates a similar activity through respiration incapacitation and apoptosis [55]. However, 21 was identified as a potential cell membrane disruptor via sterol biosynthesis inhibition [56], while 22 induces reactive oxygen species (ROS) generation. Structural activity relation (SAR) studies of high affinity protein kinase inhibitors, staurosporine-based compounds (24-27) revealed the 4th $\mathrm{C}$ methyl amine and 7 th $\mathrm{C}$ hydrogen acceptor as the cause for the reinforced activity observed in L. donovani, which had major morphological changes in the flagella pocket and plasma membrane because of signal blockage via phosphokinase (PK) inhibition.

\subsubsection{Phenolics}

As characterized by hydroxy-phenyl groups, polyphenolics are widely distributed in nature and have been isolated from different plants. In traditional medicine phenolics have received much interest in phyto-therapeutics for the treatment of ailments ranging from non-infectious to infectious diseases. These chemotypes include compounds like coumarins, flavonoids, quinones, lignans, flavone glycosides amongst others (Table 2). Flavonoids from Selaginella sellowi when tested against different forms of Leishmania revealed a pro-drug mechanism for $\mathbf{2 8}$ but an activated NO generation for $\mathbf{2 9}$ [70]. The difference in the mode of action 
Table 123 alkaloids isolated from various flora and fauna together with their $\mathrm{IC}_{50}$ and toxicity tested on some Leishmania species

Natural product source Chemical structure

\begin{tabular}{l}
$\begin{array}{l}\text { Class of } \\
\text { natural } \\
\text { product }\end{array}$ \\
\hline
\end{tabular}

product

Imidazole

28.1

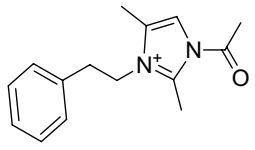

L. donovani

Low toxicity profiles to mouse macrophages

RAW 264.7 cell

lines. $>250 \mu \mathrm{M}$

Paenidigyamycin G, 1

Paenibaccillus sp. (Marine)<smiles>Cc1c(C)[n+](CCc2ccccc2)c(-c2n(CCC(C)C)c(C)c(C)[n+]2CCc2ccccc2)n1CCC(C)C</smiles>

L. major (Promastigote)

$\mathrm{MIC}=25 \mu \mathrm{M}$

Imidazole

1.90

L. donovani

(Promastigote)

References

Paenidigyamycin A, 2

Cissampelos sympodialis<smiles>COc1cc2c3c(c1O)Oc1ccc(cc1)CC1=NCCc4cc(OC)c(c(c4O)C3)OCc3ccc(cc3)-c3c(cc(OC)c(O)c31)CCN2C</smiles>

Isoquinoline

L. chasi

(Promastigote)
$\mathrm{IC}_{50}=0.056 \mu \mathrm{M}$ against human laryngeal cancer cells (HEP-2cells) and $0.067 \mu \mathrm{M}$ against human mucoepide cells (NCIH-292)

\section{Warifteine, 3}

Croton pullei var. glabrior<smiles>CC[C@H](C)C(=O)N[C@H]1CCC(=O)N(CCc2ccccc2)C1=O</smiles>

Piperidine

6.27

\section{L. amazonensis (Amastigote)}

Julocrotine, 4 
Table 1 (continued)

Natural product source

Chemical structure

Class of

$\mathrm{IC}_{50} / \mu \mathrm{g} / \mathrm{mL} \quad$ Organism tested

Toxicology

References

product

Aconitum spicatum

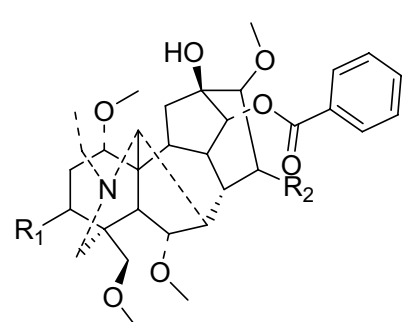

Pyrrolidine

L. major

No toxicity against MCF7,

[59]

HeLa, PC3 cancer cell

lines and 3T3 normal

fibroblast cell line at

$30 \mu \mathrm{M}$

56.0

36.1

Chasmacotine, $\mathrm{R}_{1}=\mathrm{R}_{2}=\mathrm{OAc}, 5$

Ludaconitine, $\mathrm{R}_{1}=\mathrm{OH}, \mathrm{R}_{2}=\mathrm{OH}, 6$

Helietta apiculata<smiles>COc1c2ccoc2nc2c(OC)cccc12</smiles>

Quinoline

17.3

L. donovani

[60]

Fagarine, 7<smiles>COc1c2ccoc2nc2c(OC)c3c(cc12)OCO3</smiles>

Quinoline

\section{Maculine, 8}

Thalictrum alpinum

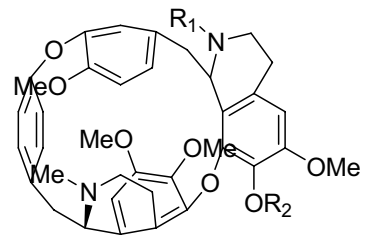

Northalrugosidine, $\mathrm{R}_{1}=\mathrm{R}_{2}=\mathrm{H}, 9$

$\begin{array}{lll}\text { Isoquinoline } & 0.639 & \text { L. donovani }\end{array}$

Thalrugosidine, $\mathrm{R}_{1}=\mathrm{Me}, \mathrm{R}_{2}=\mathrm{H}, 10$

Thalidasine, $\mathrm{R}_{1}=\mathrm{R}_{2}=\mathrm{Me}, 11$ 
Table 1 (continued)

Natural product source

Chemical structure

Class of natural

product

Trichosprum $\mathrm{sp}$

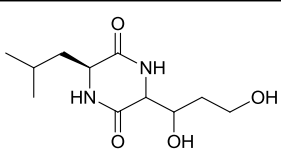

(6S)-3-(1,3- dihydroxypropyl)-6-(2-methylpropyl)piperazine-2,5-dione, 12

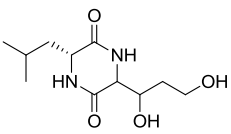

(6R)-3-(1,3- dihydroxypropyl)-6-(2-methylpropyl)piperazine-2,5-dione, 13

Piper choba<smiles>CC(C)CNC(=O)/C=C/C=C/c1ccc2c(c1)OCO2</smiles>

Piperlonguminine, 14<smiles>O=C(/C=C/C=C/c1ccc2c(c1)OCO2)N1CCCCC1</smiles>

Piperine, 15

Senna spectabilis<smiles>CC(=O)CCCCCCCCCC[C@H]1CC[C@@H](O)[C@H](C)N1</smiles>

\section{Piperidine}

24.9

L. major

(Promastigotes)

No observed lethality against J774 murine macrophage<smiles>CC(=O)CCCCCCCCCCCCC1CCC(O)C(C)N1</smiles>

Aspidosperma ramiflorum<smiles>C/C=C\C1CC2CCc3c([nH]c4ccccc34)C2CC1CC1NCCc2c1[nH]c1ccc(OC)cc21</smiles>

Indole

$\mathrm{H}-17 \alpha$ - ramiflorine $\mathrm{A}, 18$

$\mathrm{H}-17 \beta$ - ramiflorine $\mathrm{B}, 19$ 
Table 1 (continued)

Natural product source
Chemical structure

Class of natural

product

Beilschmiedia alloio-

phylla<smiles>COc1ccc2c(c1)CC1(C)C2=C2C=C(O)C=C2CCN1C</smiles>

quinoline

2.95

$[65$

2-hydroxy-9-methoxyaporphine, 20

Berberis vulgaris

Isoquinoline 2.10

2.90

L. major

L. tropica

(Promastigotes)

Observed toxicity agains murine macrophage was at $9.18 \mu \mathrm{M}$

[66]<smiles>COc1ccc2cc3[n+](cc2c1OC)CCc1cc2c(cc1-3)OCO2</smiles>

References

\section{Berberine, 21}

Piper longum<smiles>CC(C)CNC(=O)CCCCCC/C=C/c1ccc2c(c1)OCO2</smiles>

Amide

9.12

\section{(p. donovani
(promastigotes)}

Test against J774A.

cell line indicated a

high cytotoxicity at $5.05 \pm 0.64 \mu \mathrm{g} / \mathrm{mL}$. 393
L. donovani (amastigotes)
[67]

Piperlongumide, 22

Spongia sp. and Ircinia

$$
\text { sp. }
$$

(Marine)
Indole

9.6<smiles>OCCc1c[nH]c2ccccc12</smiles>

Toxicity profile against mammalian L6 cells was

Tryptophol, 23 
Table 1 (continued)

Natural product source

Chemical structure

Class of natural

product

Streptomyces sanyensis

(Marine)<smiles>CNC1C[C@H]2O[C@](C)(C1OC)n1c3ccccc3c3c4c(c5c6ccccc6n2c5c31)C(=O)NC4=O</smiles>

7-oxostaurosporine, 24

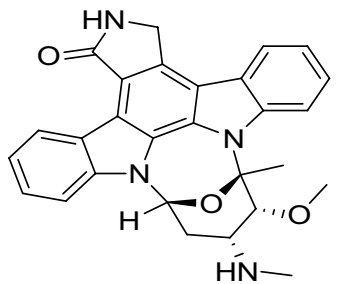

Staurosporine, 25<smiles>CO[C@H]1C(=O)C[C@H]2OC1(C)n1c3ccccc3c3c4c(c5c6ccccc6n2c5c31)CNC4=O</smiles>

40-demethyl-40oxostaurosporine, 26<smiles>COC1=C(C)O[C@H]2C[C@]1(OC)n1c3ccccc3c3c4c(c5c6ccccc6n2c5c31)C(=O)NC4</smiles>

$\mathrm{IC}_{50} / \mu \mathrm{g} / \mathrm{mL} \quad$ Organism tested

Toxicology

References
0.0075 L. ama
ensis
(promast (promastigotes)

Indolocarbazole
0.0012
L. donovani (promastigotes)
0.0002
L. amanzon-
ensis (amastigotes)

L. amanzon-

8.74

Indolocarbazole

0.004 (promastigotes)

0.0224

L. donovani (promastigotes)

L. amanzon-

ensis (amastigotes)

$\begin{array}{cll}\begin{array}{c}\text { Indolocar- } \\ \text { bazole }\end{array} & 0.037 \quad \begin{array}{l}\text { L. amanzon- } \\ \text { ensis } \\ \text { (promastigotes) }\end{array} \\ & >0.089 \quad \begin{array}{l}\text { L. donovani } \\ \text { (promastigotes) }\end{array} \\ & \begin{array}{l}\text { L. amanzon- } \\ \text { ensis } \\ \text { (amastigotes) }\end{array}\end{array}$

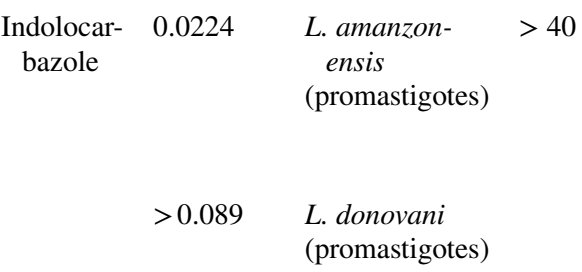

Streptocarbazole B, 27 
of these two flavonoids may be due to their conformational orientations. Similar investigations to understand the possible cause of apoptosis induced by $\mathbf{3 0}$ and $\mathbf{3 1}$ suggested a mitochondrial dysfunction with no influence on ROS [71]. However, evidence from suicidal action of some quercetin analogues have also indicated iron chelation, arginase inhibition, and topoisomerase II intercalation as possible mechanisms [72]. From the same Nectandra genus, inhibitory activity of $\mathbf{3 4}$ and $\mathbf{4 3}$ have been fully elucidated. Results indicated an inactivation of exacerbatory immunogens with reduced calcium levels and depolarized mitochondria potential [73]. Studies with similar compounds against melanoma cells indicated an apoptosis process confirming the depolarization activity [74]. Deciphering the exact mechanism underpinning the leishmanicidal action of isolated compounds from Connarus seberosus, it was revealed that defects in the mitochondria and plasma membrane structure with the evidence of lipid accumulation were caused by $\mathbf{5 5}$ and 56 [75]. Comparing 58 and its 3-O-methyl analog, 59, to rosmarinic acid (based on the shared catechol nucleus), their potential mode of action is suggested as inhibition of reactive oxygen species [76, 77]. 75 as a chemo-preventive agent acts by reducing inflammatory symptoms by suppressing NF- $\kappa \mathrm{B}$ expression and other pro-inflammatory factors including iNOS, COX-2, TNF- $\alpha$, IL-1 $\beta$, and IL-6 [78]. Compound 74 emulates an apoptosis induced suicidal mechanism which involves DNA fragmentation, inhibition of inflammation cytokines and the activation of caspases with downstream effects on gene transcriptional process [79]. Structural similarities of anti-inflammatory coumarins with 74 precludes a similar mechanism of action [80]. From the isolates of Arrabidaea brachypoda only $\mathbf{6 7}$ altered organelle structure and function by attenuating cytoplasm puncturing and golgi apparatus swellings [81].

\subsubsection{Terpenes and terpenoids}

Another group of secondary metabolites with interesting anti-parasitic activities are terpenes. Ultrastructural changes of 79 in phenotypic screenings indicated mitochondrial blebs and lipid deformities $[100,101] .80$ isolated from essential oils of Tetradenia riparia were found to distort promastigote structure especially the fate of its chromatin followed by an apoptosis process which is suspected to be caused by caspase activation [102, 103] (Tables 3 and 4).

Halogenated terpenes $\mathbf{7 2}$ and $\mathbf{8 3}$ from Laurencia dendroidea which only differ primarily in a double bond character also targets the same organelle via redox perturbation [104, 105]. The natural product $\mathbf{8 7}$ from Vanillosmopsis arborea show promising activity through apoptosis induction characterized by mitochondrial dysfunction and oxidative stress [106]. Similar mode of action was reported for 87 isolated from Tunisia chamomile essential oil against $L$. amazonensis and L. infantum [107]. Effects of clerodone terpenes, 88, 89 and $\mathbf{9 0}$ from the stem bark of Croton cajucara have been shown to obstruct ROS protection via trypanothione reductase inhibition [108].

Interest in marine natural products which led to the evaluation of marine terpenes like pentacyclic triterpene $\mathbf{9 2}$, which exhibited an anti-inflammatory action with enhanced levels of $\mathrm{T}$ cells and Th1 cytokines when compared to its control [109].

Elucidation of the exact mechanism of action of four triterpenes from the roots of Salvia deserta showed that despite the strong antioxidant capacity of $\mathbf{9 3}$, it also kill parasites by inhibiting isopentenyl diphosphate condensation with the major target being farnesyl diphosphate synthase [110]. Studies to also understand the molecular basics of $\mathbf{9 4}$ shows a similar action like 80, but fragmentation of DNA strands has been described for diterpene 95 and 96 [111, 112]. Inhibition of oxidative pathways particularly IFN- $\gamma$-related signaling by similar diterpenoid quinones isolated from the roots of Salvia officinalis has also been shown to prevent disease proliferation and further protecting the host specie [113]. Recent studies in estimating the role of the energy production in the form of ATP in Leishmania with acyl phloroglucinol derivatives has revealed $\mathbf{9 7}$ as a mitochondria complex II/III inhibitor [114].

Like terpenes which are formed by the head to tail condensation of isoprene units, terpenoids (terpenes with oxygen-containing functional group) also represent a unique group of natural products with high functionalization and promising pharmacological activity. Isolation of six germacranolides from the leaves and stems of the Calea species have shown promising activities against $L$. donovani and $L$. amazonensis $[115,116]$. Among them morphological assessment studies with $\mathbf{1 0 0}$ and $\mathbf{1 1 1}$ indicated alterations in the nucleus and mitochondria describing an apoptosis like process through the mitosis motor downregulation pathway [115]. Due to the similar core structure shared with germacra-1(10),11(13)-dien-12,6-olide a similar mechanism is envisaged for its counterpart $\mathbf{1 0 4}$ by aiding in generating ROS complementing the elucidated apoptosis process. The natural product $\mathbf{1 0 6}$ shares same structural core therefore may possess similar mode of action in addition to the inhibition of thiol-antioxidant enzymes [117]. Interestingly, 106 and its iso-conformer have also been disclosed to induce a proinflammatory inhibition via the NF-KB pathway [118]. On the other hand, $\mathbf{1 1 0}$ and $\mathbf{1 2 5}$ have also exerted multi-spectral activities including suppression of cell proliferation modulators and upregulation of microbicidal NO species [119].

\subsubsection{Steroids}

Steroids are a class of natural or synthetic organic compounds with three six membered rings fused with a five 


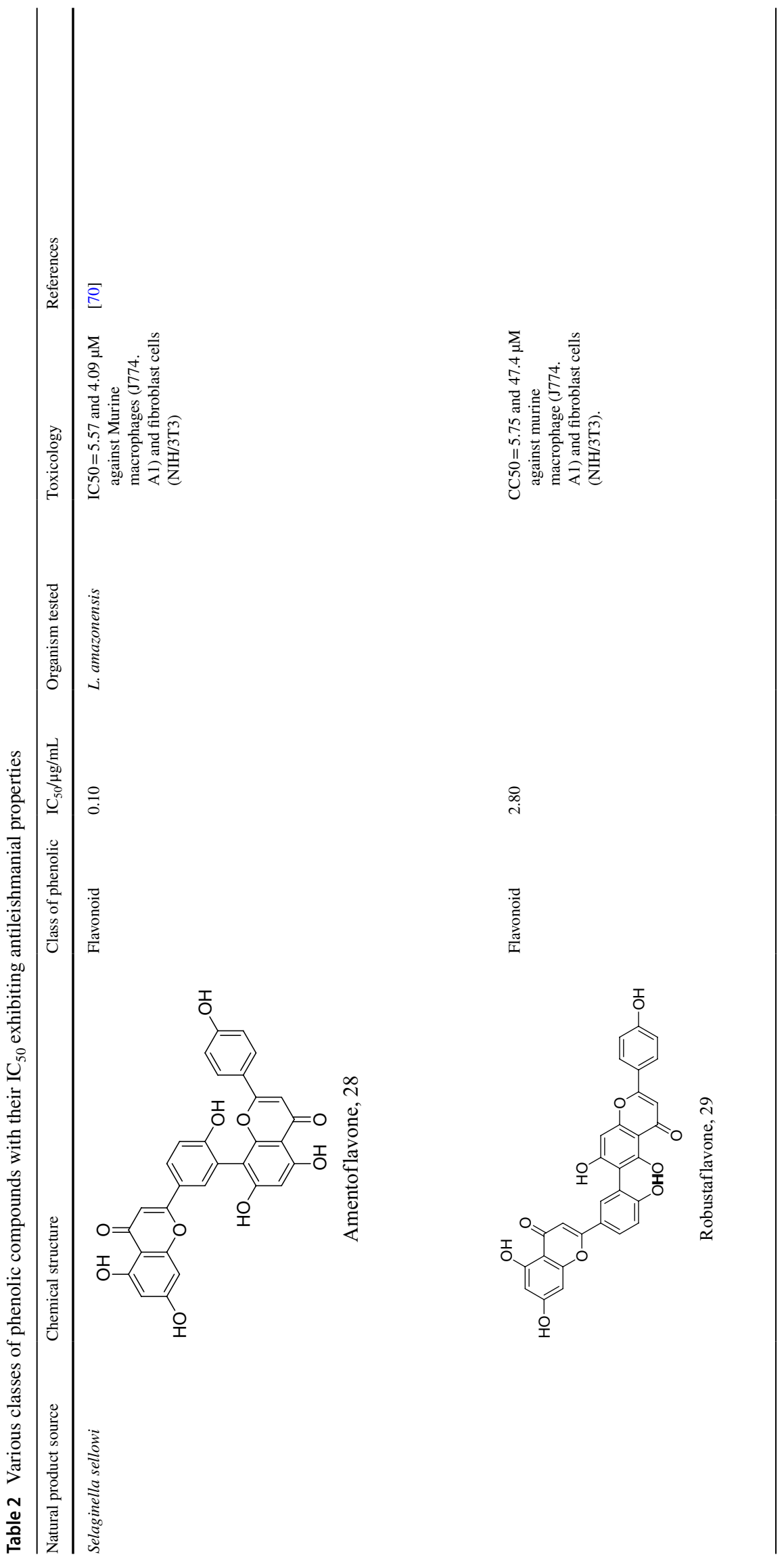




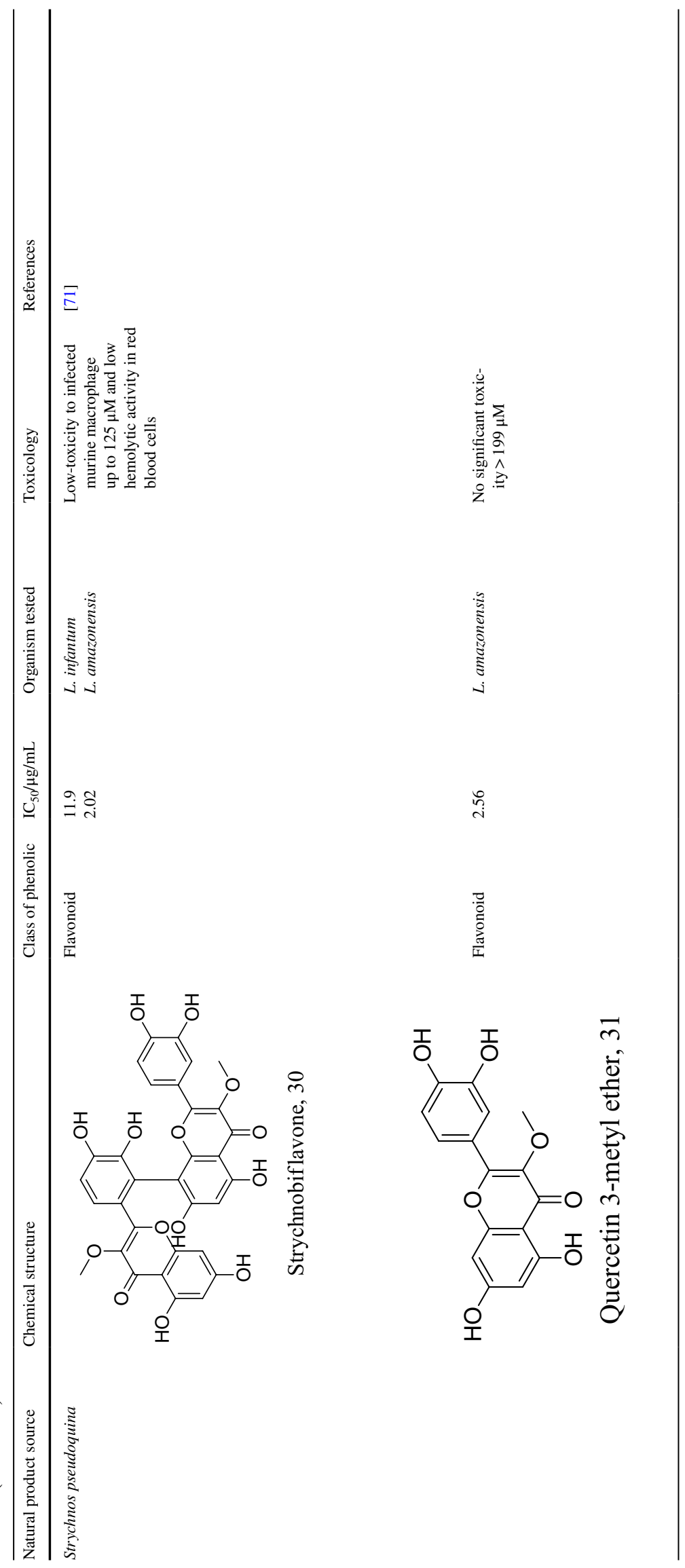




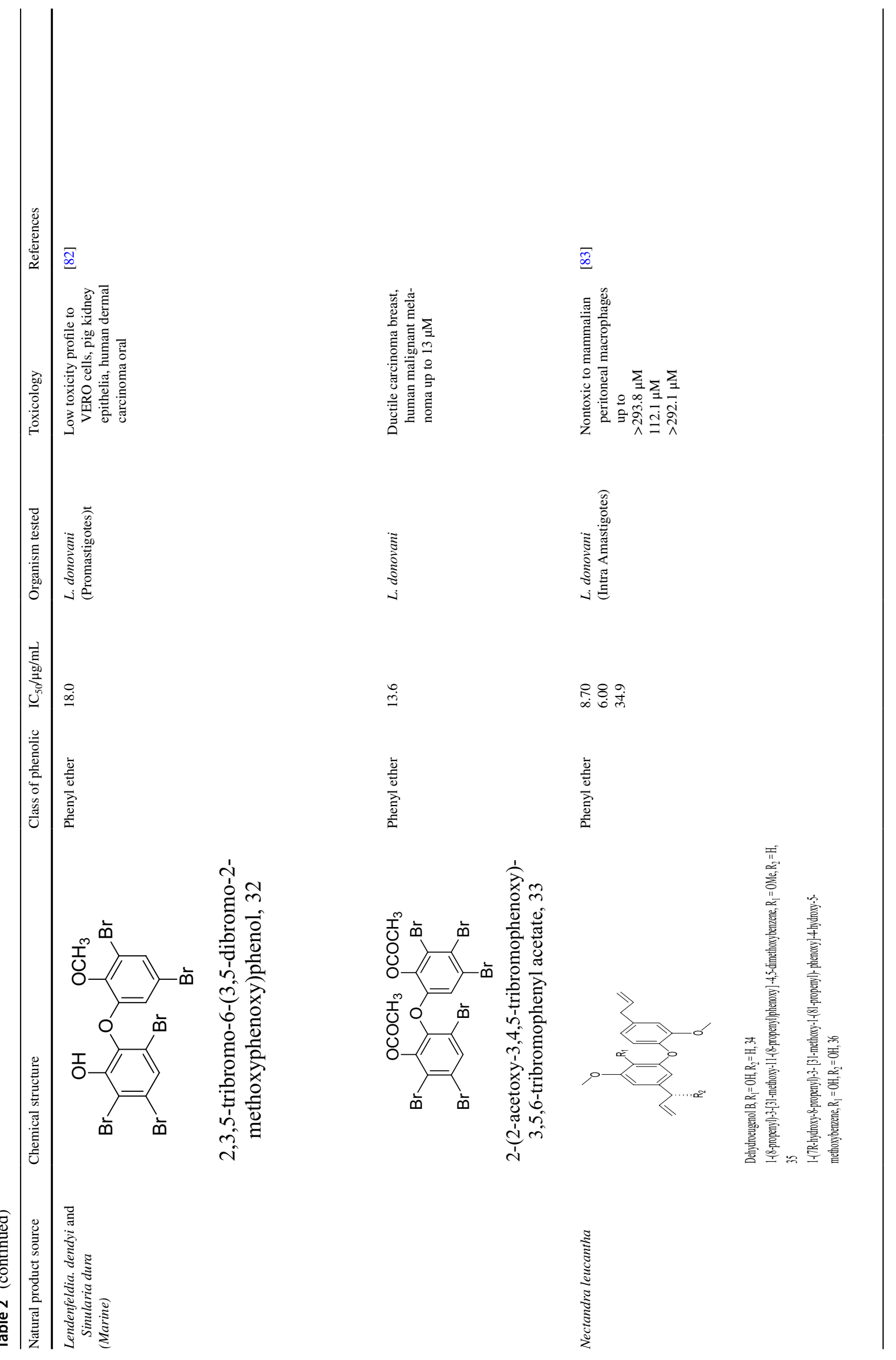




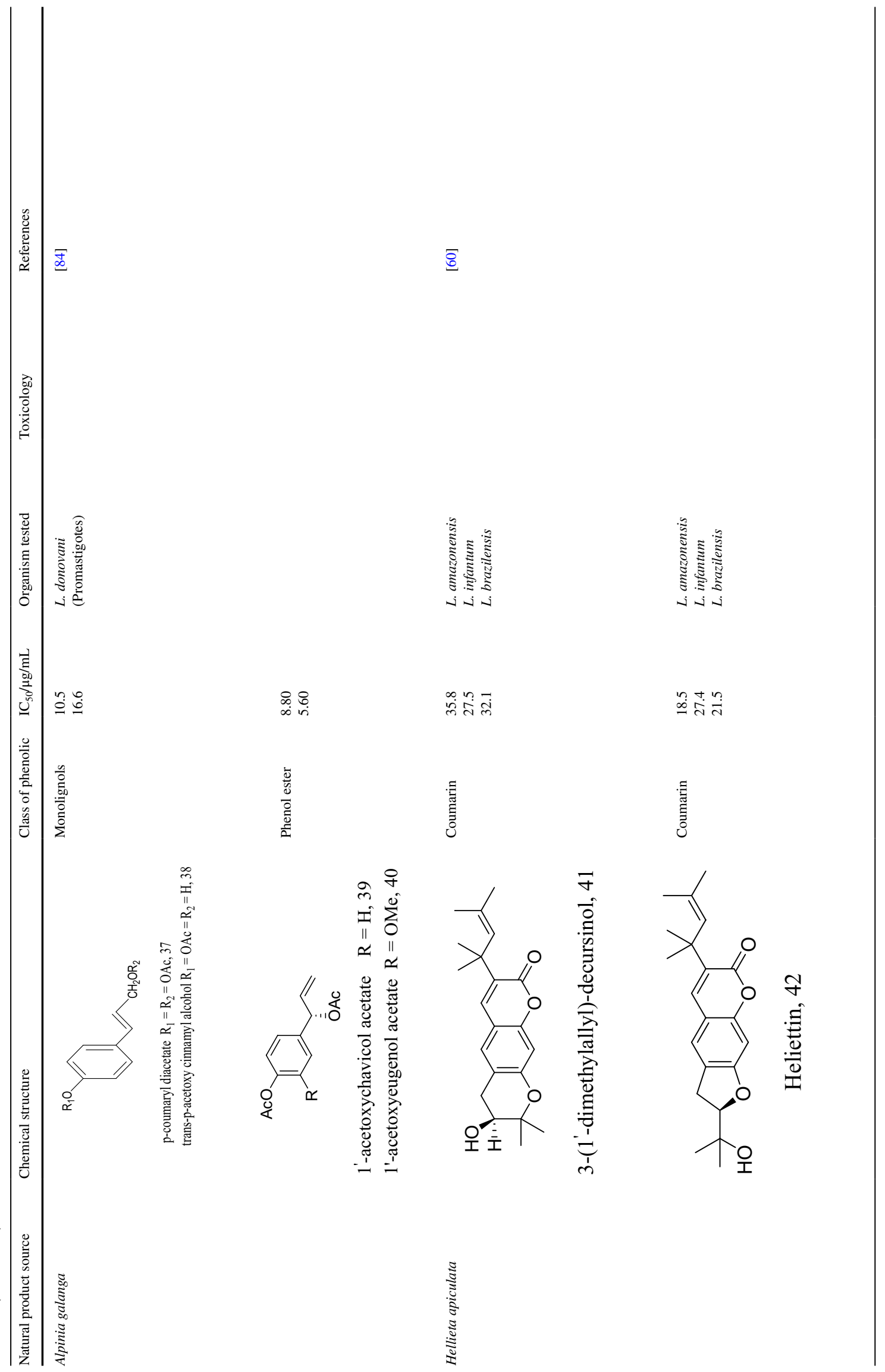




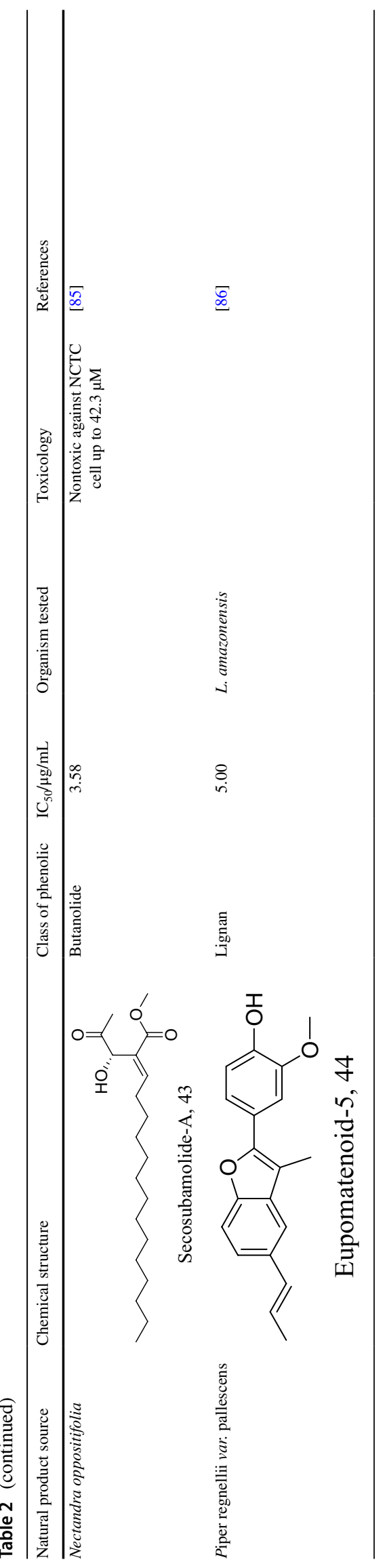

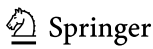




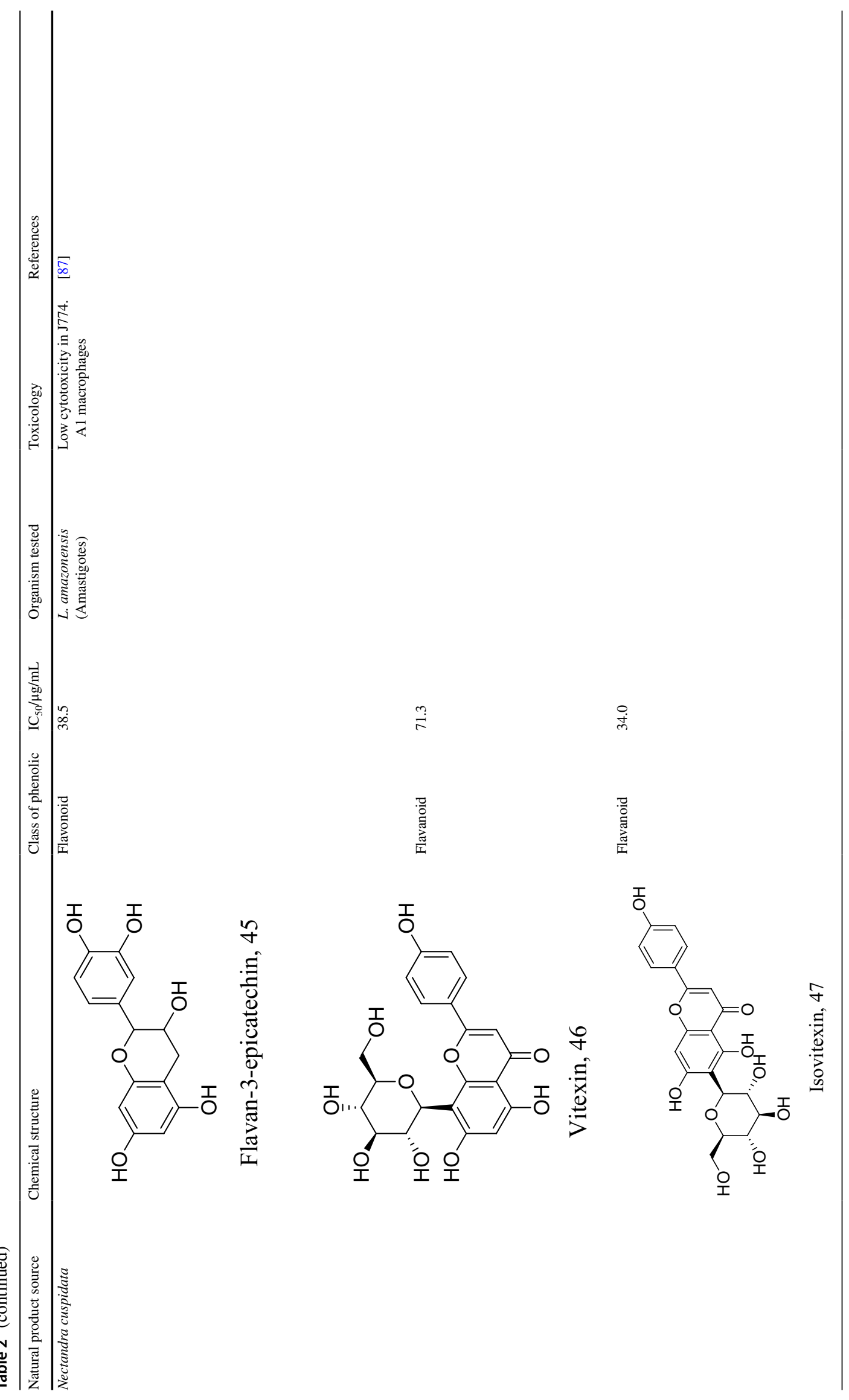




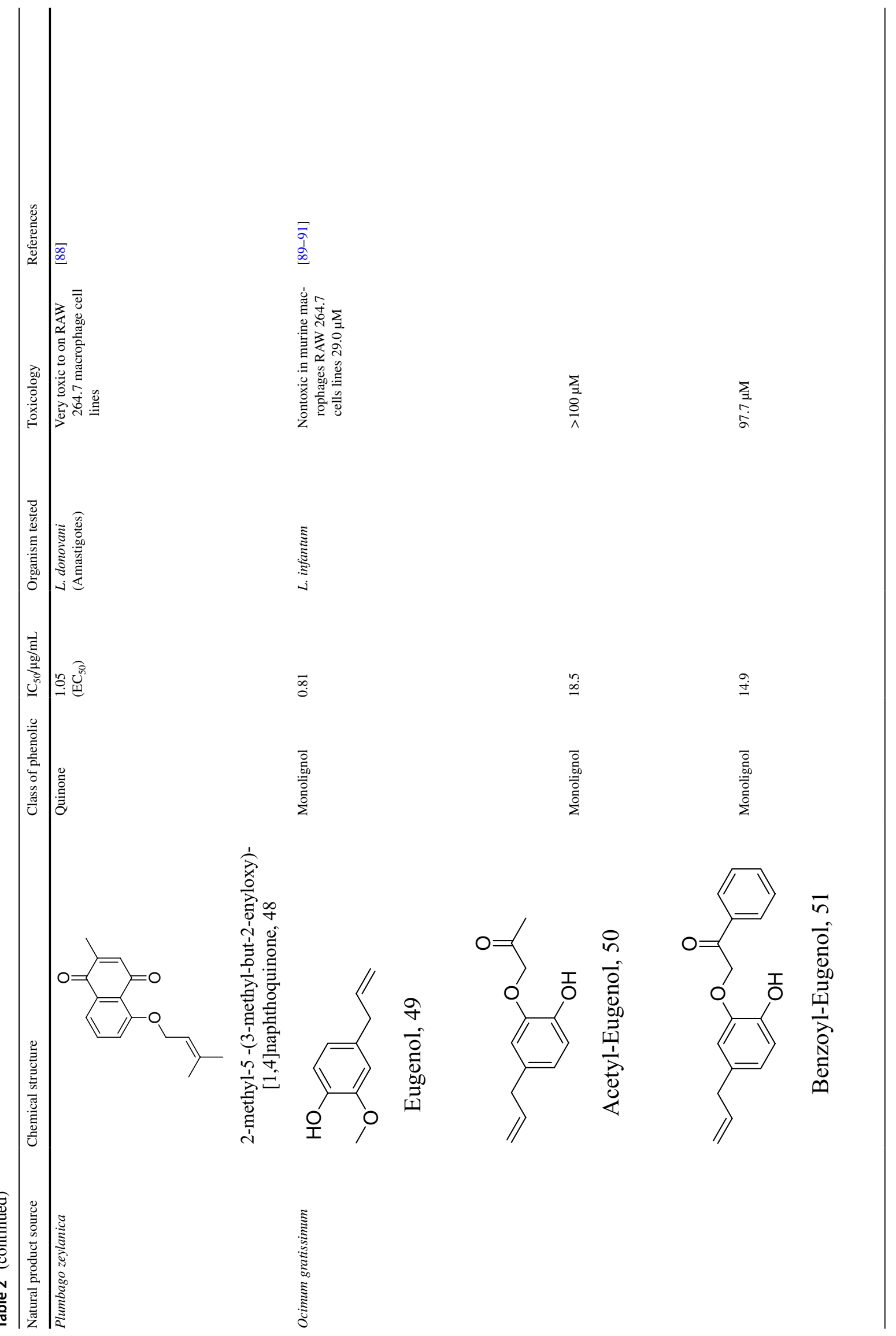

包 Springer 


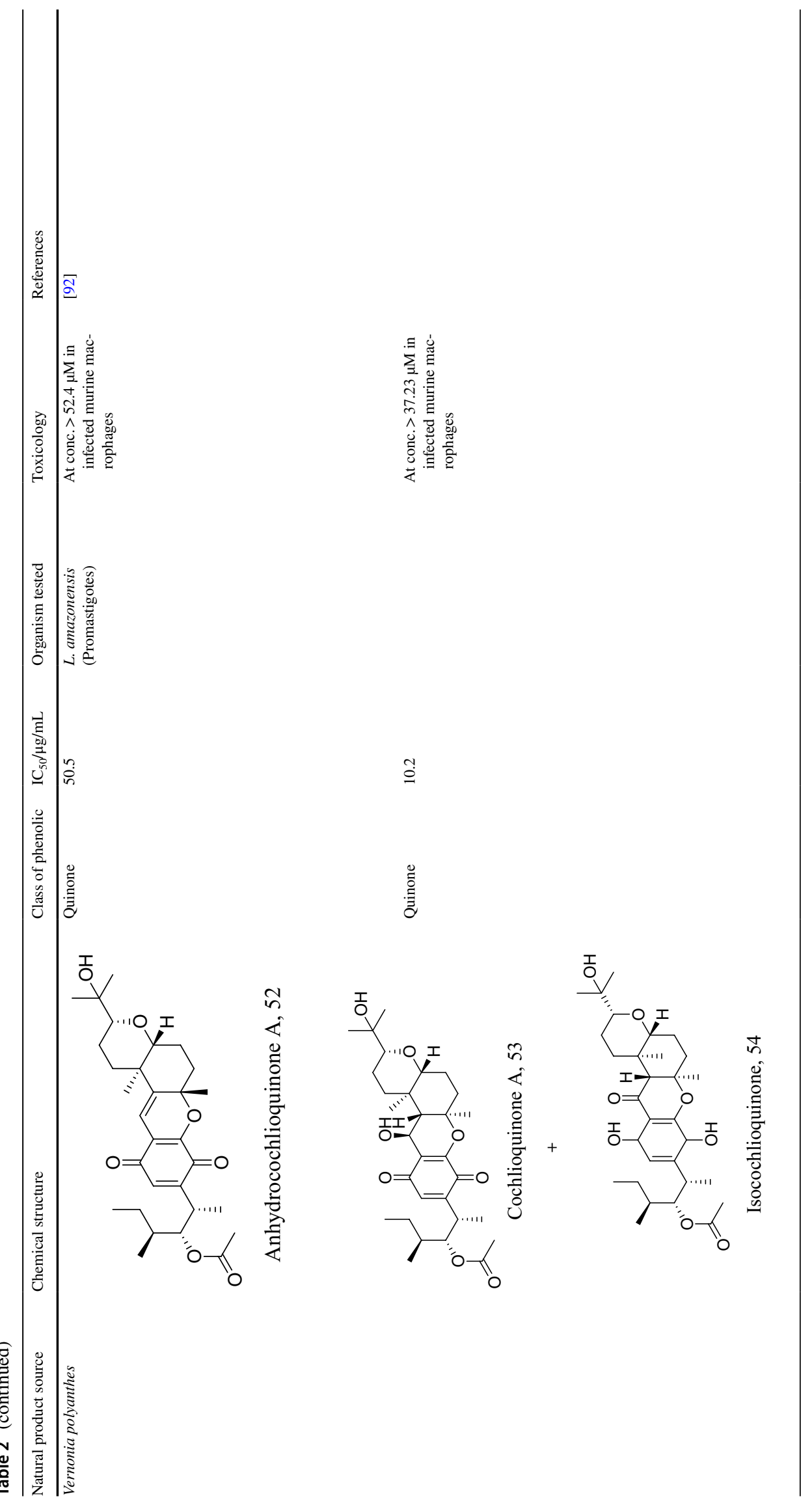




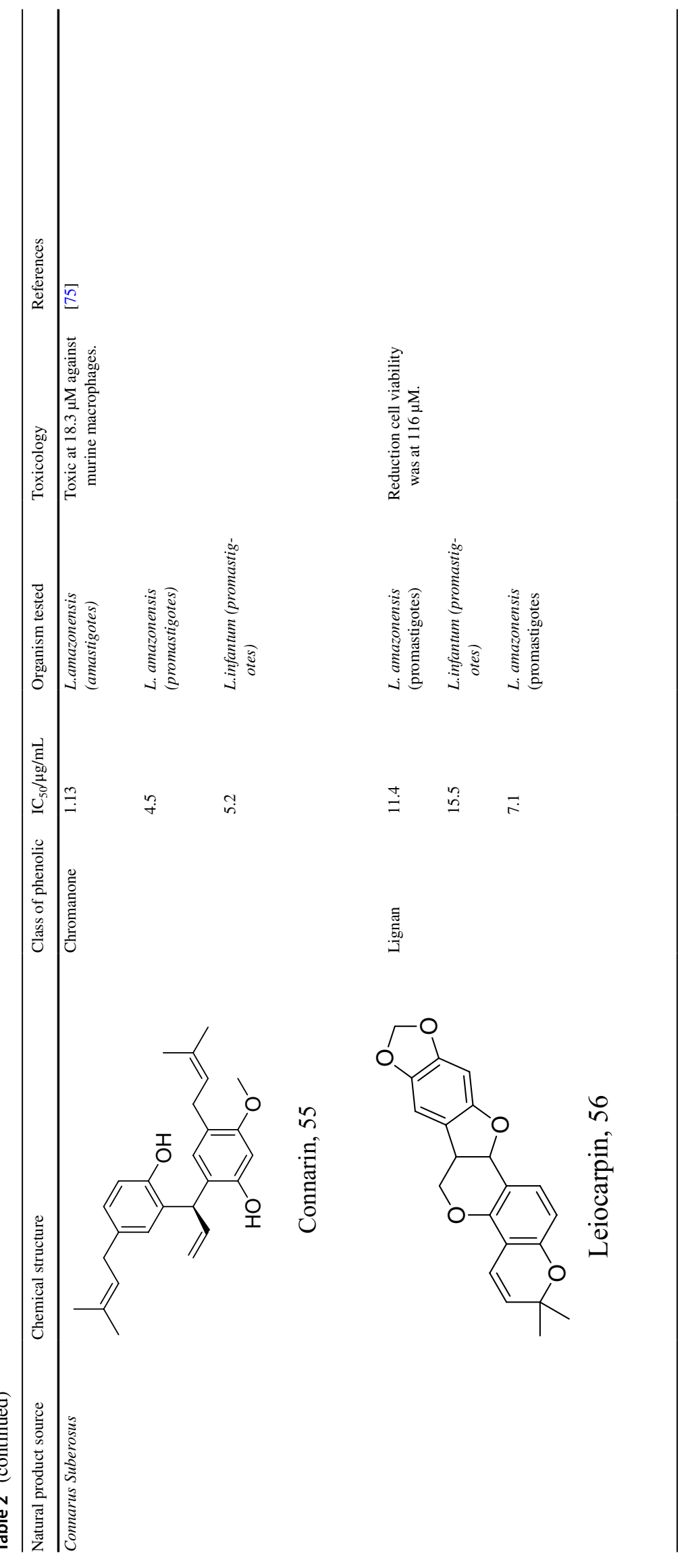




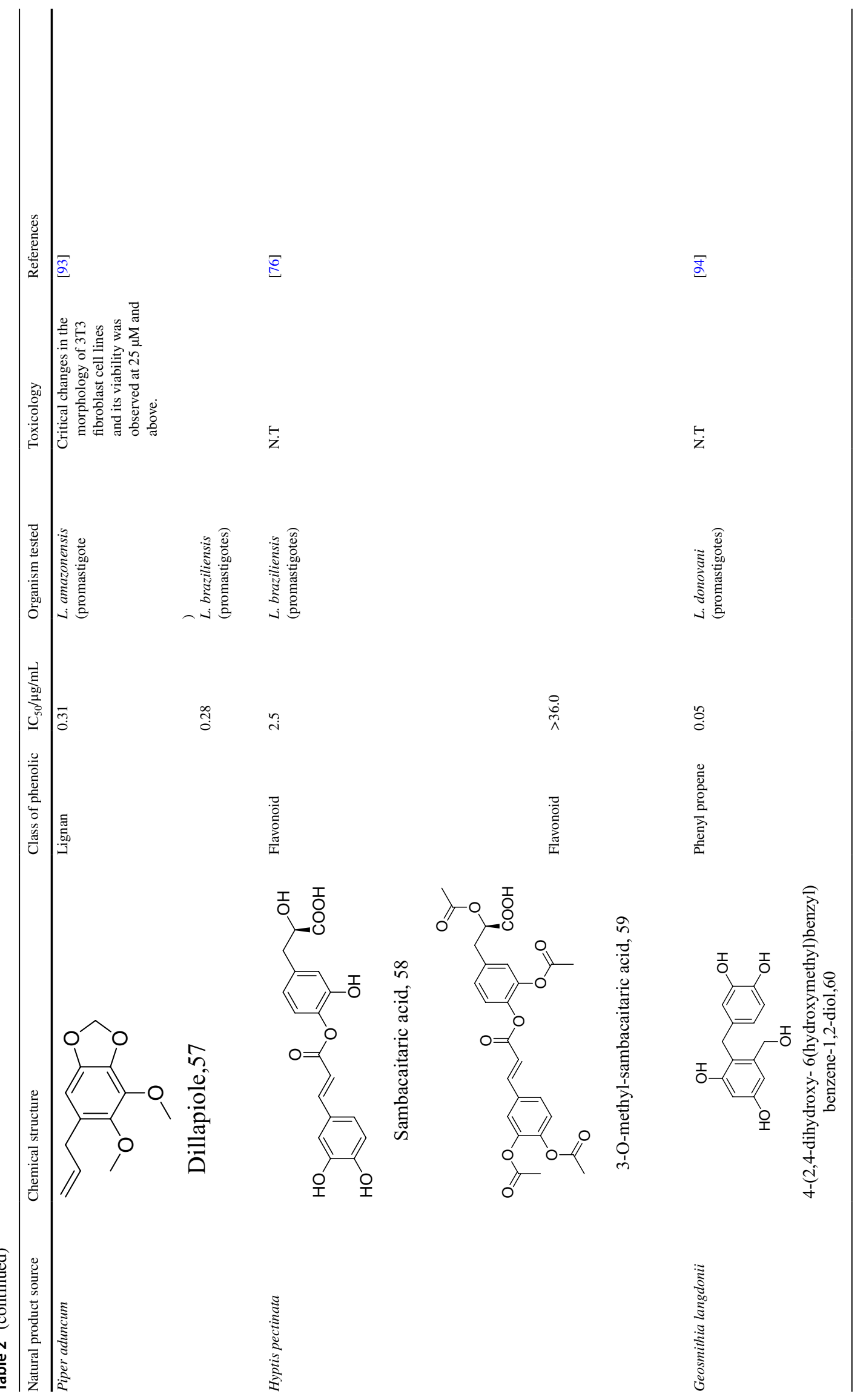




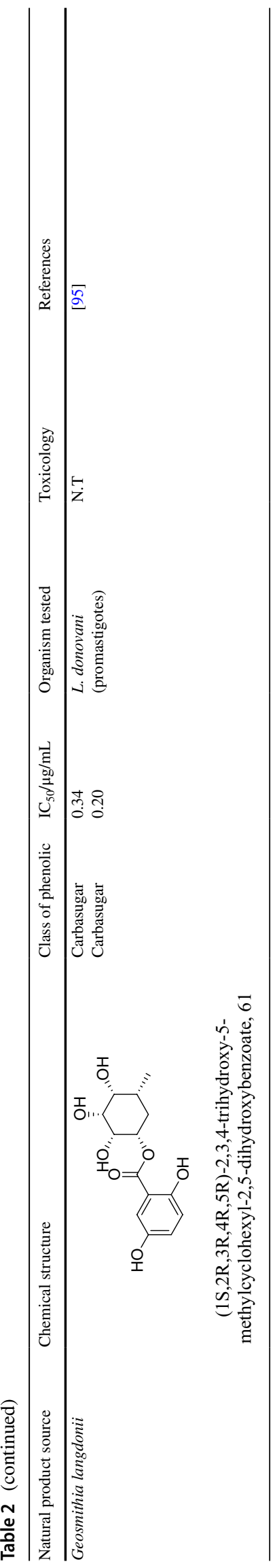

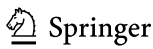




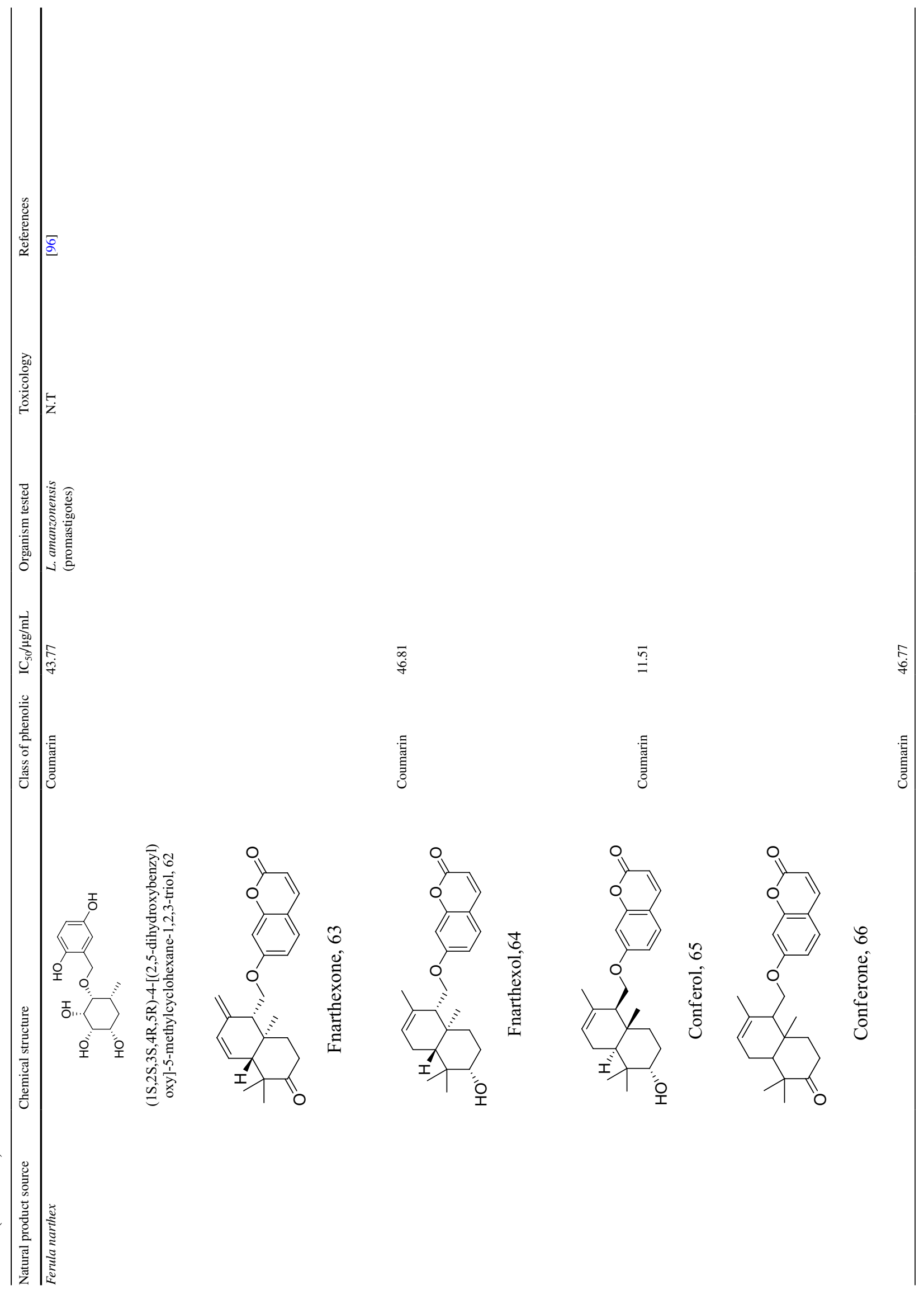




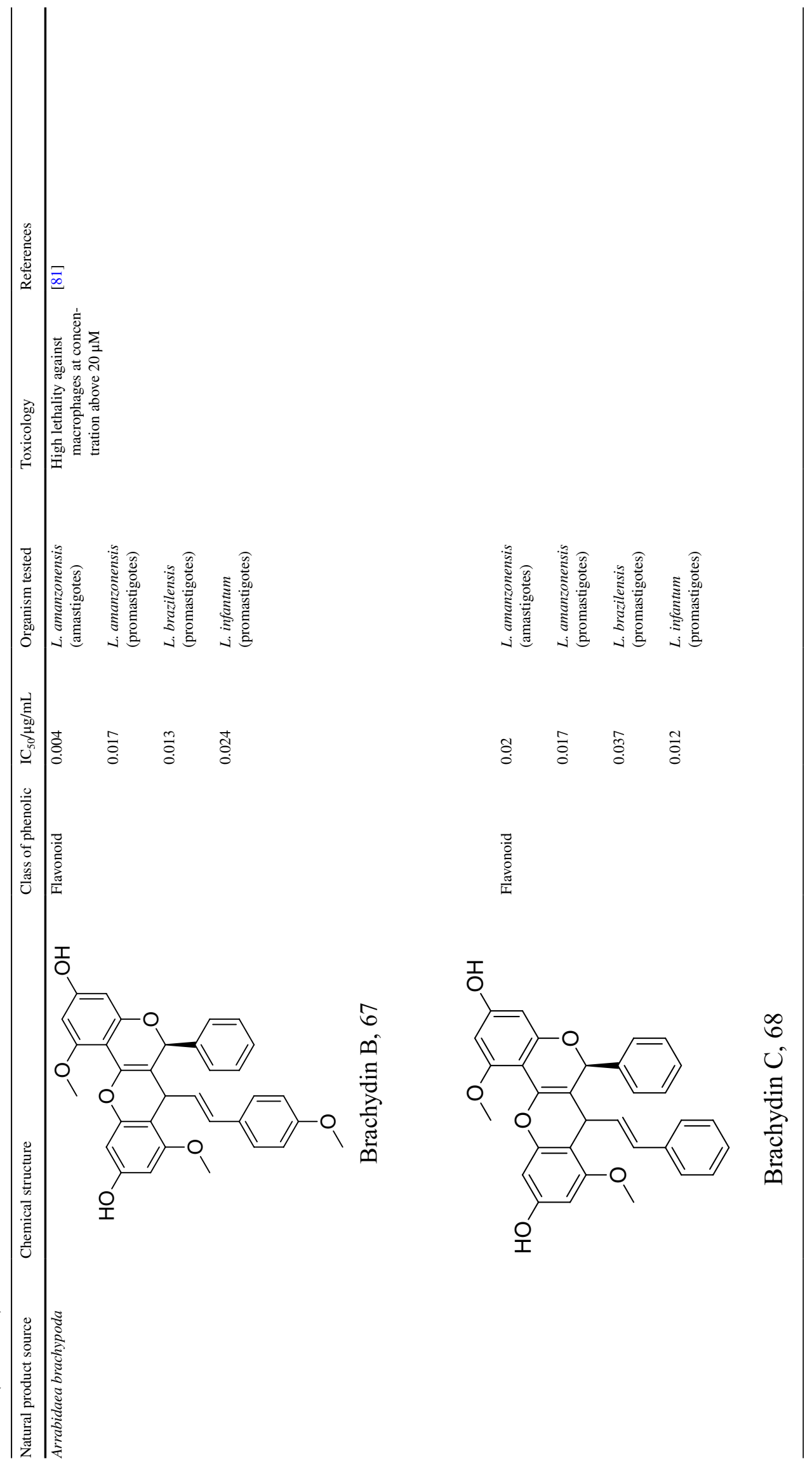




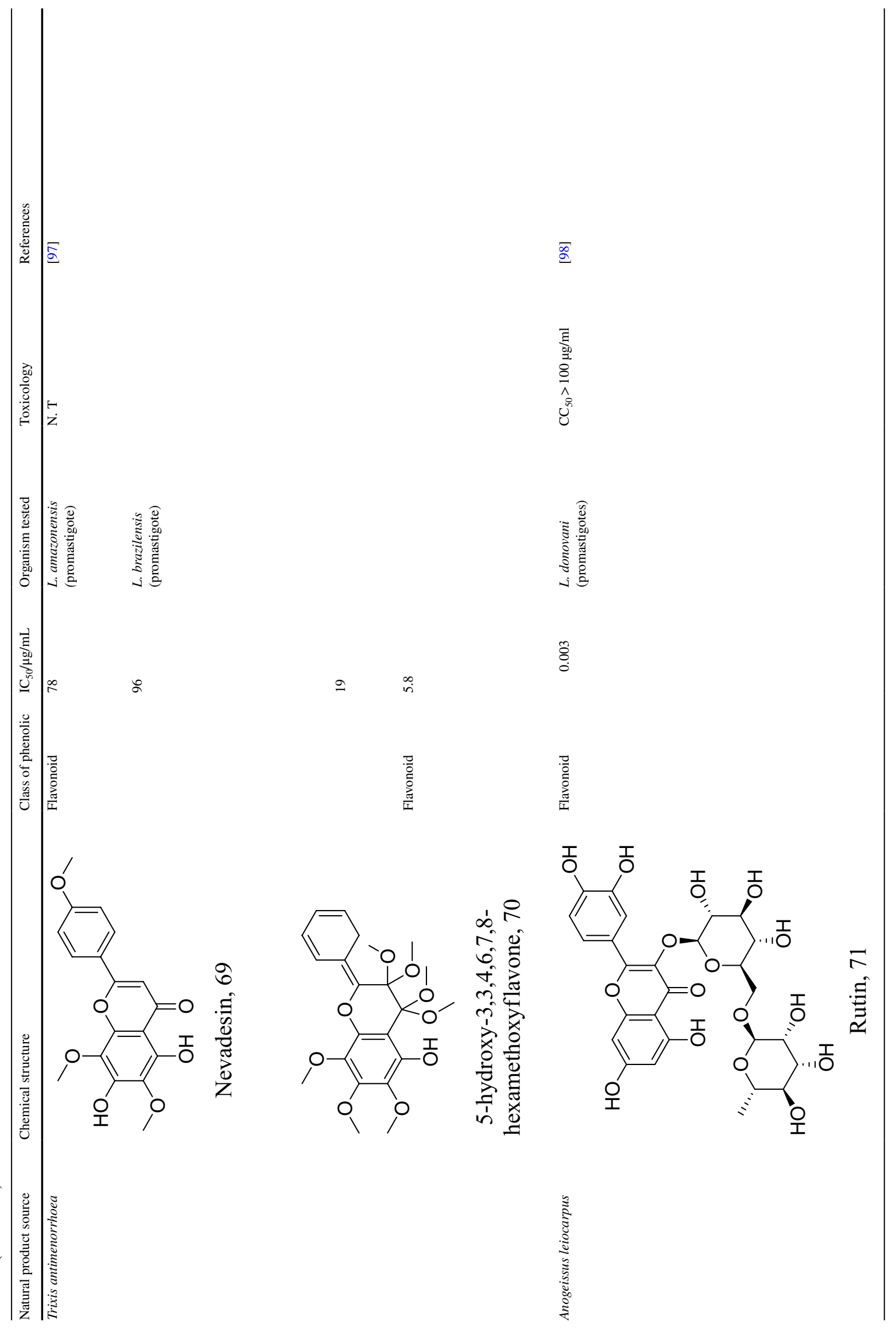




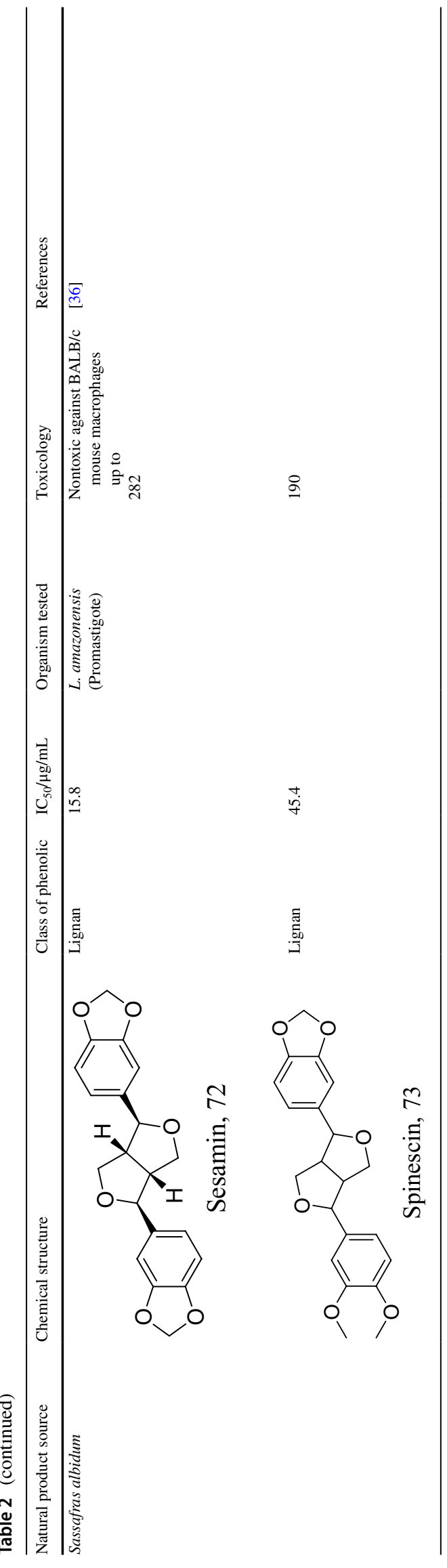




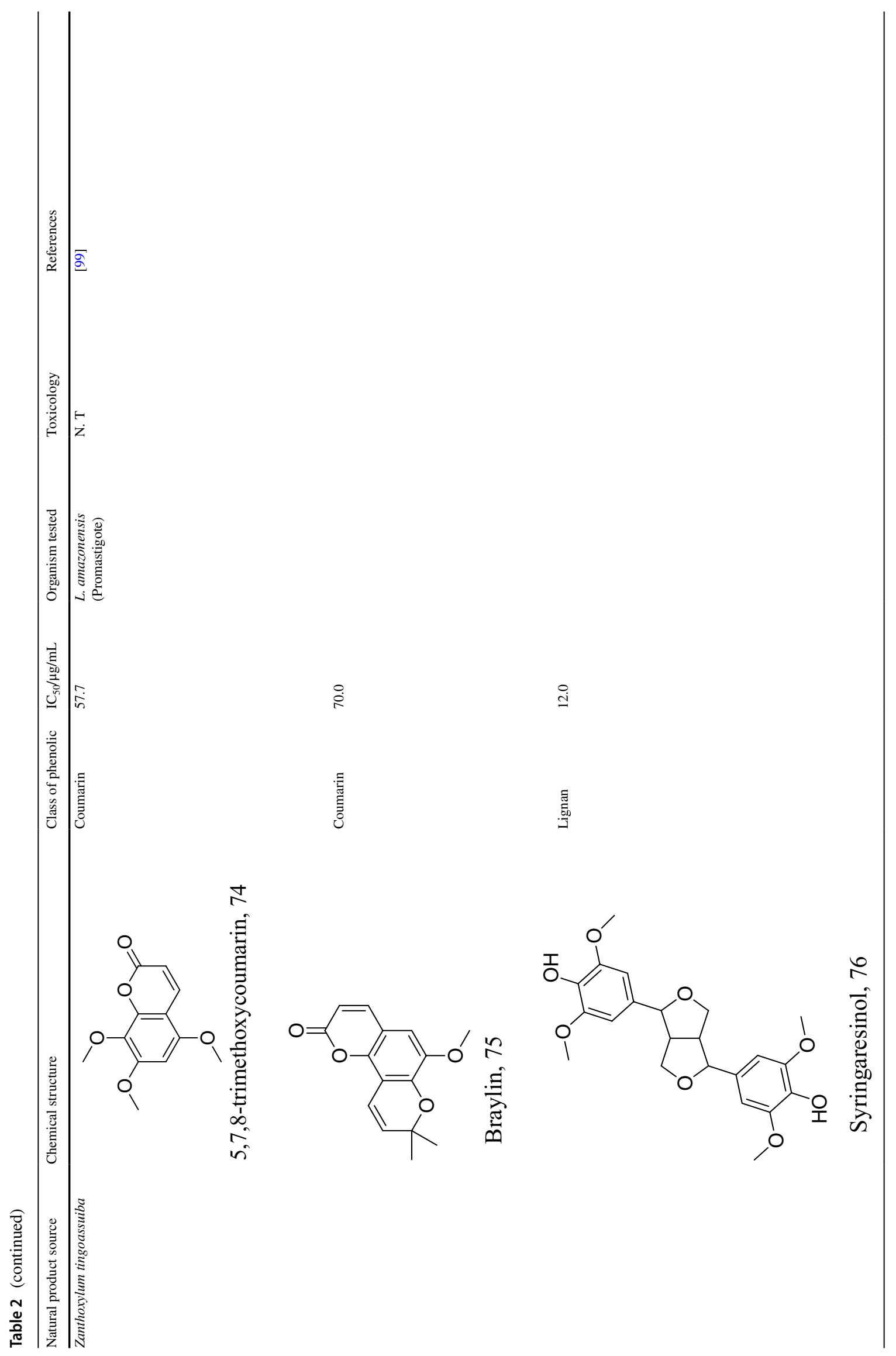


Table 3 Various classes of terpenes and terpenoids with their $\mathrm{IC}_{50}$ exhibiting antileishmanial properties

\begin{tabular}{|c|c|c|c|c|c|c|}
\hline $\begin{array}{l}\text { Natural product } \\
\text { source }\end{array}$ & Chemical structure & $\begin{array}{l}\text { Class of natural } \\
\text { product }\end{array}$ & $\mathrm{IC}_{50} / \mu \mathrm{g} / \mathrm{mL}$ & $\begin{array}{l}\text { Organism } \\
\text { tested }\end{array}$ & Toxicology & References \\
\hline Parinari excelsa & & Triterpenoid & 0.05 & $\begin{array}{l}\text { L. donovani } \\
\text { (amastigotes) }\end{array}$ & $\begin{array}{l}\text { Cell viability assay } \\
\text { with L6 cell lines } \\
\text { revealed the lethal } \\
\text { concentration at } \\
73.5 \mu \mathrm{g} / \mathrm{mL}\end{array}$ & {$[120]$} \\
\hline
\end{tabular}

\section{6-hydroxylupane-1,20(29)-} dien-3-one, 77

Morinda lucida<smiles>COC(=O)C1=COCC2C1C=CC21OC(=O)/C(=C/c2ccc(O)c(OC)c2)C1O</smiles>

Molucidin, 78

\section{Canistrocarpus cervicornis} (Marine)<smiles>C=C1CC[C@@H](OC(C)=O)C2CC=C3[C@](C)(CC[C@@]3(O)C(C)C)C[C@]12O</smiles>

(4R,9S, 14S)-4a-acetoxy-9b, 14a-dihydroxydolast1(15),7-diene, 79

Tetradenia riparia<smiles>C=C1C(=O)OC2C1CCC(=C)C1CCC(C)(O)C12</smiles>

[121] promastig otes)

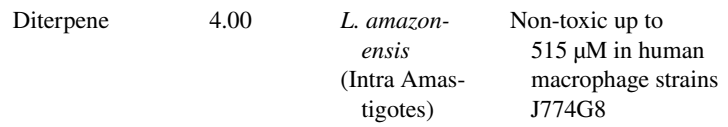

Diterpene

4.00

L. amazon ensis (Intra Amastigotes)

Non-toxic up to $515 \mu \mathrm{M}$ in human macrophage strains J774G8

[100]

\begin{tabular}{|c|c|c|c|}
\hline Sesquiterpene & 2.45 & $\begin{array}{l}\text { L. amazon- } \\
\text { ensis } \\
\text { (Promastig- } \\
\text { otes) }\end{array}$ & $\begin{array}{l}\text { high toxicity } \\
\text { against mouse } \\
\text { peritoneal mac- } \\
\text { rophages }=1.69 \mu \mathrm{M}\end{array}$ \\
\hline
\end{tabular}

6,7-Dehydroroyleanone, 80 
Table 3 (continued)

\begin{tabular}{|c|c|c|c|c|c|c|}
\hline $\begin{array}{l}\text { Natural product } \\
\text { source }\end{array}$ & Chemical structure & $\begin{array}{l}\text { Class of natural } \\
\text { product }\end{array}$ & $\mathrm{IC}_{50} / \mu \mathrm{g} / \mathrm{mL}$ & $\begin{array}{l}\text { Organism } \\
\text { tested }\end{array}$ & Toxicology & References \\
\hline $\begin{array}{l}\text { Laurencia den- } \\
\text { droidea } \\
\text { (Marine) }\end{array}$ & & Sesquiterpene & 10.8 & $\begin{array}{l}\text { L. amazon- } \\
\text { ensis } \\
\text { (Intra Amas- } \\
\text { tigotes) }\end{array}$ & $\begin{array}{l}\mathrm{CC}_{50} \text { in macrophages } \\
\text { and lymph nodes in } \\
\text { amastigotes cervical } \\
\text { BALB/c mice } \\
160.2 \text { and } 172.8 \mu \mathrm{M}\end{array}$ & [123] \\
\hline
\end{tabular}

Triquinane, 81<smiles>C=C1C[C@H](O)[C@H](Br)C(C)(C)C1(CC)CC(Cl)=C(C)C</smiles>

Elatol, 82<smiles>C=C1C[C@@H](O)[C@H](Br)C(C)(C)C12CCC(C)(Br)C(Cl)C2</smiles>

Sesquiterpene $\quad 1.50$

112.9 and $120.2 \mu \mathrm{M}$

Sesquiterpen $\quad 1.62$
133.5 and $139.3 \mu \mathrm{M}$

Obtusol, 83 
Table 3 (continued)

\begin{tabular}{|c|c|c|c|c|c|c|}
\hline $\begin{array}{l}\text { Natural product } \\
\text { source }\end{array}$ & Chemical structure & $\begin{array}{l}\text { Class of natural } \\
\text { product }\end{array}$ & $\mathrm{IC}_{50} / \mu \mathrm{g} / \mathrm{mL}$ & $\begin{array}{l}\text { Organism } \\
\text { tested }\end{array}$ & Toxicology & References \\
\hline $\begin{array}{c}\text { Combretum } \\
\text { leprotum }\end{array}$ & & Triterpene & 3.30 & $\begin{array}{l}\text { L. amazon- } \\
\text { ensis } \\
\text { (Promastig- } \\
\text { otes) }\end{array}$ & $\begin{array}{l}\text { Non-toxic against } \\
\text { mouse peritoneal } \\
\text { macrophages }\end{array}$ & {$[124] \mathrm{t}$} \\
\hline
\end{tabular}

3, 6, 16-trihydroxylup-20(29)-ene, 84<smiles>C=C(C)[C@@H]1CC[C@]2(C)C(=O)C[C@]3(C)[C@@H](CC[C@@H]4[C@@]5(C)CCC(=O)C(C)(C)[C@@]5(C)C(=O)C[C@]43C)[C@H]12</smiles>

3, 6, 16 -trioxolup-20(29)-ene, 85<smiles>C=C(C)[C@H]1CC[C@]2(C)C(O)C[C@]3(C)[C@@H](CC[C@@H]4[C@@]5(C)CC[C@H](O)C(C)(C)[C@@]5(C)C(=O)C[C@]43C)[C@H]12</smiles>

Triterpene $\quad 5.80$

3,16-dihydroxy-6oxolup-20(29)-ene, 86

Vanillosmopsis arborea<smiles>CC(C)=CCCC(C)(O)C1CC=C(C)CC1</smiles>

$\alpha$-Bisabolol, 87

$\begin{array}{cccc}\text { Sesquiterpene } 10.7 & \begin{array}{c}\text { L. amazon- } \\ \text { ensis } \\ \text { (Amastigotes) }\end{array} & \begin{array}{c}\text { Low cytotoxicity } \\ \text { to macrophage } \\ \text { J777.G8 cell lines } \\ \end{array} & \begin{array}{ll}451 \mu \mathrm{M}\end{array}\end{array}$

[106] 
Table 3 (continued)

\begin{tabular}{|c|c|c|c|c|c|c|}
\hline $\begin{array}{l}\text { Natural product } \\
\text { source }\end{array}$ & Chemical structure & $\begin{array}{l}\text { Class of natural } \\
\text { product }\end{array}$ & $\mathrm{IC}_{50} / \mu \mathrm{g} / \mathrm{mL}$ & $\begin{array}{l}\text { Organism } \\
\text { tested }\end{array}$ & Toxicology & References \\
\hline Croton cajucara & & Diterpene & 20.0 & $\begin{array}{l}\text { L. amazon- } \\
\text { ensis } \\
\text { (Axenic Amas- } \\
\text { tigotes) }\end{array}$ & & [108] \\
\hline
\end{tabular}

trans-dehydrocrotonin, 88<smiles></smiles>

trans-crotonin, 89<smiles>CC(=O)O[C@H]1CC[C@]2(C)C3CC[C@H]4C(=CCC5(C(=O)O)CCC(C)(C)C[C@@H]45)[C@@]3(C)CC[C@@H]2C1(C)C</smiles>

Acetyl aleuritolic acid, 90

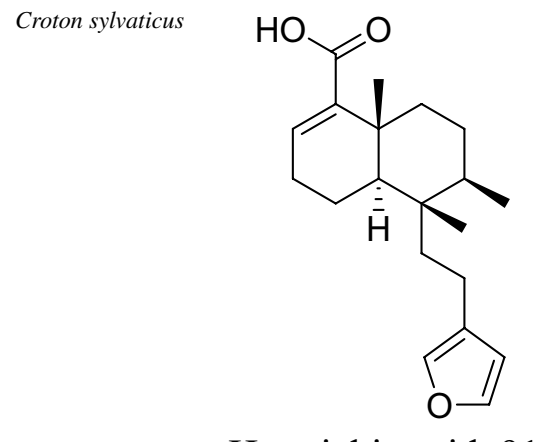

Harwickic acid, 91
Diterpene $\quad 41.4$

Triterpene $\quad 58.3$

$\begin{array}{cccc}\text { Diterpenoid } & 10.0 & \begin{array}{l}\text { L. major } \\ \text { (Promastig- } \\ \text { otes) }\end{array} & \begin{array}{c}\text { Observed toxicity was } \\ \text { low at } 247.83 \mu \mathrm{M}\end{array}\end{array}$


Table 3 (continued)

\begin{tabular}{llllll}
\hline $\begin{array}{l}\text { Natural product } \\
\text { source }\end{array}$ & Chemical structure & $\begin{array}{l}\text { Class of natural } \\
\text { product }\end{array}$ & $\mathrm{IC}_{50} / \mu \mathrm{g} / \mathrm{mL}$ & $\begin{array}{l}\text { Organism } \\
\text { tested }\end{array}$ & Toxicology \\
\hline Sterculia villosa & $\begin{array}{c}\text { Triterpenoid } \\
\text { (Intracellular } \\
\text { Amastig- } \\
\text { otes) }\end{array}$ & 15.0 & $\begin{array}{c}\text { N.T } \\
{[126]}\end{array}$ &
\end{tabular}

Lupeol, 92

Salvia deserta<smiles>CC(C)c1cc2c(cc1O)[C@]1(C)CCC[C@H](C)[C@H]1CC2</smiles>

Taxodione, 93<smiles>CC(C)C1=CC2=CC(=O)[C@@H]3C(C)(C)CCC[C@]3(C)C2=C(O)C1=O</smiles>

Ferruginol, 94<smiles>[R]C1C[C@]2(C)[C@@H](C)CCC[C@]2(C)C2=C1C(=O)C(C(C)C)=C(O)C2=O</smiles>

7-O-Acetyl Horminone, $\mathrm{R}=\mathrm{OAc}, 95$

Horminone, $\mathrm{R}=\mathrm{H}, 96$ $\begin{array}{llll}\text { Diterpenoid } & 0.46 & \text { L. donovani N. T }\end{array}$

[35] 
Table 3 (continued)

$\begin{aligned} & \text { Natural product } \\ & \text { source }\end{aligned}$ Chemical structure
$\begin{gathered}\text { Garcinia acha- } \\ \text { chairu }\end{gathered}$

Myrsinoic acid B, 98 
Table 3 (continued)

\begin{tabular}{|c|c|c|c|c|c|c|}
\hline $\begin{array}{l}\text { Natural product } \\
\text { source }\end{array}$ & Chemical structure & $\begin{array}{l}\text { Class of natural } \\
\text { product }\end{array}$ & $\mathrm{IC}_{50} / \mu \mathrm{g} / \mathrm{mL}$ & $\begin{array}{l}\text { Organism } \\
\text { tested }\end{array}$ & Toxicology & References \\
\hline Calea zacatechichi & O & $\begin{array}{l}\text { Sesquiterpene } \\
\text { Lactone }\end{array}$ & 1.89 & L. donovani & & [116] \\
\hline
\end{tabular}

Sesquiterpene $\quad 0.771$ 
Table 3 (continued)

\begin{tabular}{|c|c|c|c|c|c|c|}
\hline $\begin{array}{l}\text { Natural product } \\
\text { source }\end{array}$ & Chemical structure & $\begin{array}{l}\text { Class of natural } \\
\text { product }\end{array}$ & $\mathrm{IC}_{50} / \mu \mathrm{g} / \mathrm{mL}$ & $\begin{array}{l}\text { Organism } \\
\text { tested }\end{array}$ & Toxicology & References \\
\hline $\begin{array}{l}\text { Tanacetum parthe- } \\
\text { nium }\end{array}$ & & $\begin{array}{l}\text { Sesquiterpene } \\
\text { Lactone }\end{array}$ & 2.60 & $\begin{array}{l}\text { L. amazon- } \\
\text { ensis } \\
\text { (promastig- } \\
\text { otes) }\end{array}$ & $\begin{array}{l}\text { Low toxicity towards } \\
\text { J774G8 cells }\end{array}$ & [128] \\
\hline
\end{tabular}

Parthenolide, 105

Plumeria bicolor<smiles>C/C=C1/C(=O)O[C@]23C=C[C@H]4C(C(=O)OC)=CO[C@H](O[C@H]12)C4=C3</smiles>

Plumericin, 106<smiles>C/C=C1\C(=O)O[C@]23C=C[C@H]4C(C(=O)OC)=CO[C@H]5O[C@H]2C4C153</smiles>

Isoplumericin, 107

$$
\begin{array}{lll}
\begin{array}{l}
\text { Monoterpene } \\
\text { lactone }
\end{array} & 0.409 & \begin{array}{l}
\text { L. donovani } \\
\text { (Amastigotes) }
\end{array}
\end{array} \mathrm{CC}_{50}=20.6 \mu \mathrm{M}
$$


Table 3 (continued)

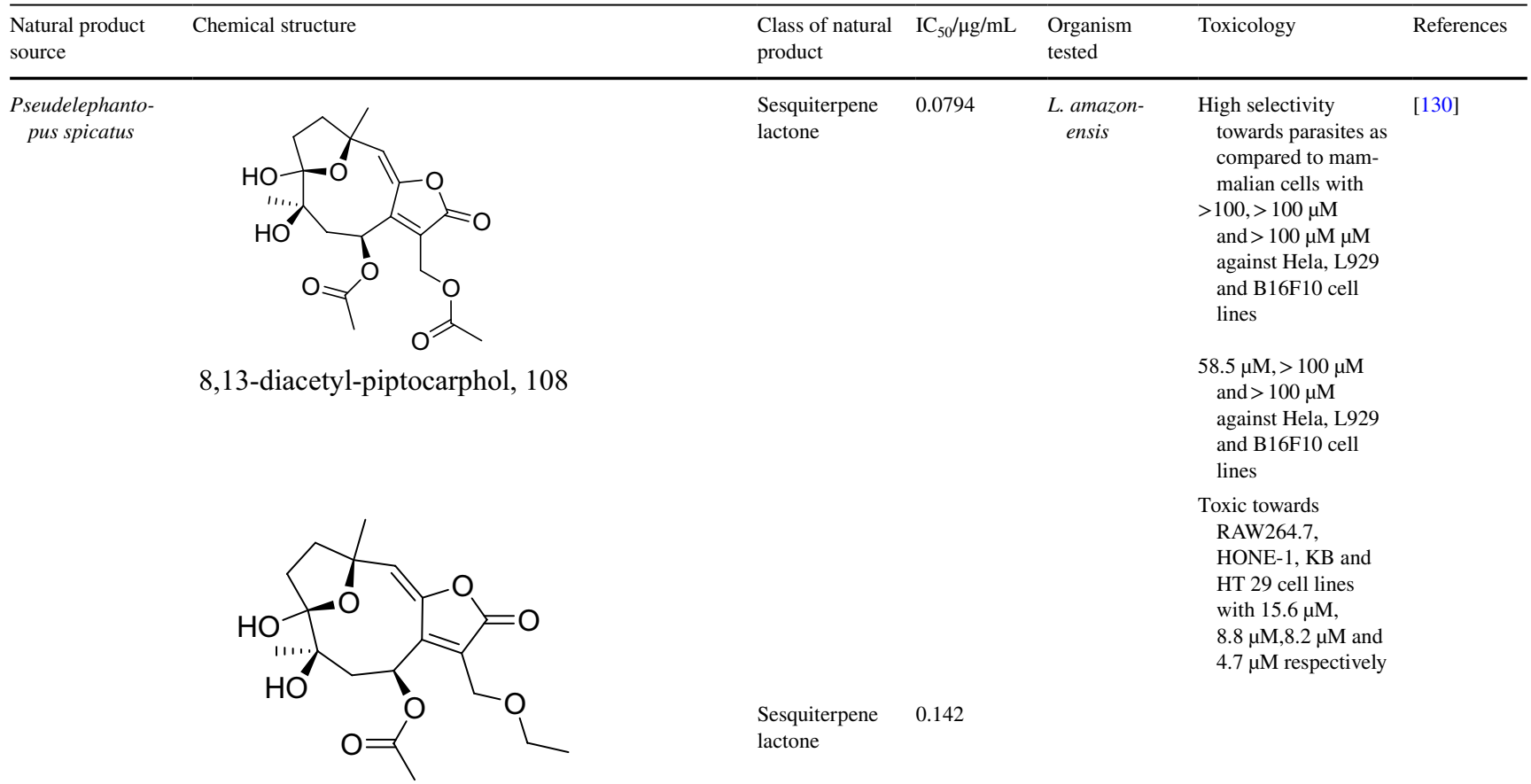

8-acetyl-13-O-ethyl-piptocarphol, 109<smiles>C[C@H]1CC[C@]2(C(=O)O)CC[C@]3(C)C(=CC[C@H]4[C@@]3(C)CC[C@]3(C)C(C)(C)[C@H](O)CC[C@]43C)[C@H]2[C@@H]1C</smiles>

Triterpenoid $\quad 0.451$

Ursolic acid, 110 
Table 3 (continued)

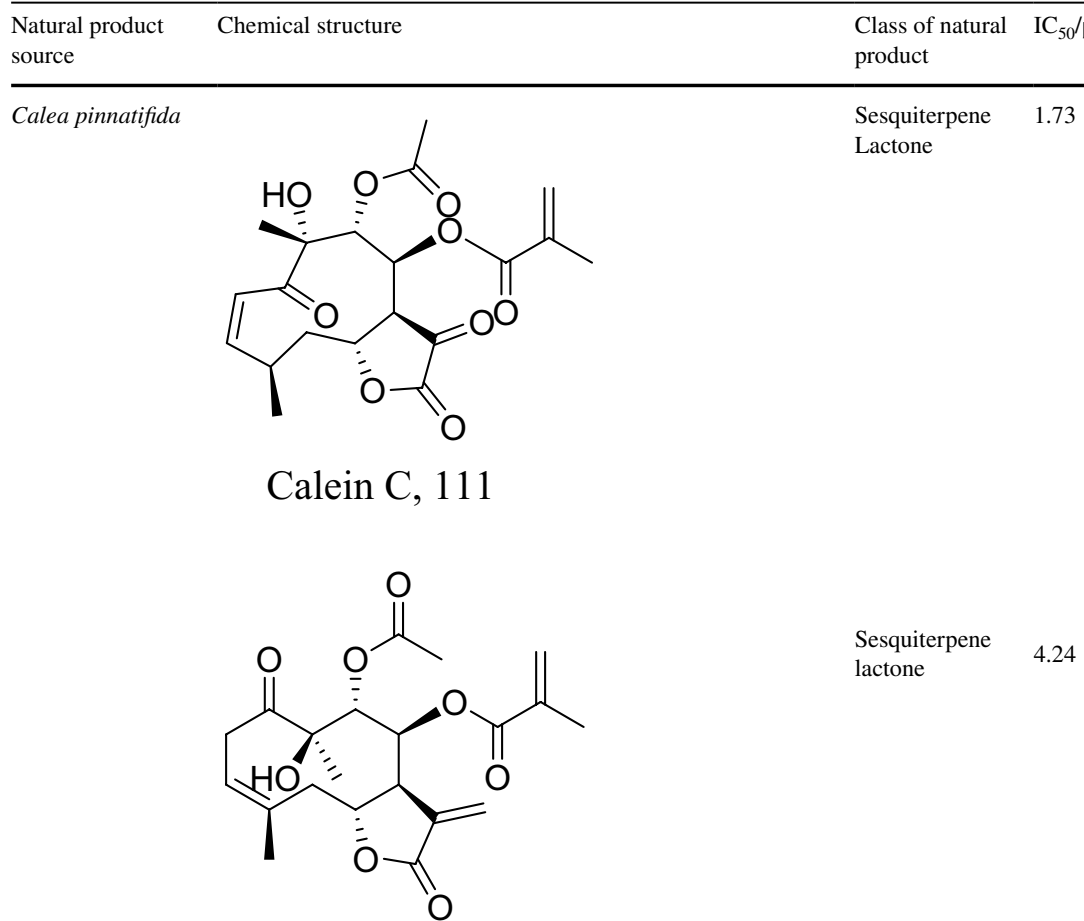

Calealactone C, 112 
Table 3 (continued)

$\begin{aligned} & \text { Natural product } \\ & \text { source }\end{aligned}$
$\begin{aligned} & \text { Spongia sp. and } \\ & \text { Ircinia sp. } \\ & \text { (Marine) }\end{aligned}$

$11 \beta$-acetoxyspongi-12-en-16-one,113

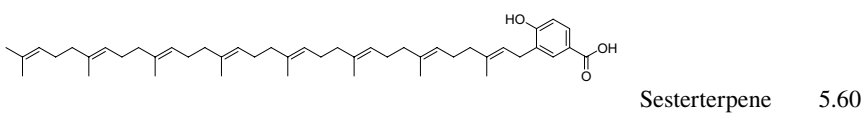

127

4-hydroxy-3-octaprenylbenzoic acid, 114<smiles>C/C(=C\CCc1ccco1)C[C@H](O)C[C@@H](C)CCCc1ccco1</smiles>

Furospongin-1, 115

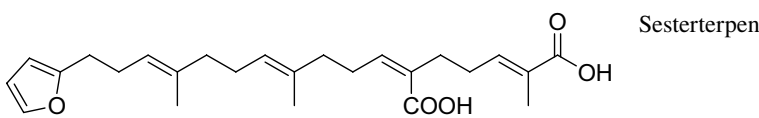

Demethylfurospongin-4, 116

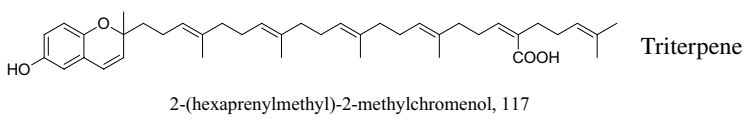

-(hexaprenylmethyl)-2-methylchromenol, 117<smiles>CC(C)=CCC/C(C)=C/CC/C(C)=C/CC/C(C)=C/CCc1ccco1</smiles>

Furospinulosin-1, 118

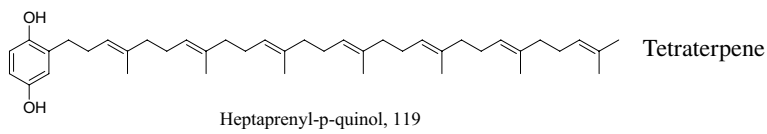

oxicity profile against mammalian L6 cells was
References

[68] 
Table 3 (continued)

\begin{tabular}{|c|c|c|c|c|c|c|}
\hline $\begin{array}{l}\text { Natural product } \\
\text { source }\end{array}$ & Chemical structure & $\begin{array}{l}\text { Class of natural } \\
\text { product }\end{array}$ & $\mathrm{IC}_{50} / \mu \mathrm{g} / \mathrm{mL}$ & $\begin{array}{l}\text { Organism } \\
\text { tested }\end{array}$ & Toxicology & References \\
\hline Baccharis tola & & Diterpenoid & 4.60 & L. brazilensis & $\begin{array}{l}\text { All compounds } \\
\text { showed high cyto- } \\
\text { toxicity in human } \\
\text { U937 macro phages } \\
\text { with values lower } \\
\text { than } 347 \mu \mathrm{M}\end{array}$ & [131] \\
\hline
\end{tabular}

Ent-beyer-15-en-18-ol, 120<smiles>CC12C=CC3(CCC4C(C)(CO)CCCC4(C)C3CC1)C2</smiles>

Ent-beyer-15-en-19-ol, 121

Jatropha muitifida<smiles>C=C1[C@@H](C)C[C@H]2[C@@H](C)[C@H]2CC[C@](C)(O)/C=C2/C(=O)C(C)=C[C@@]12O</smiles>

14-deoxy-1 $\beta$-hydroxy-4(4E)jatrogrossidentadione, 122

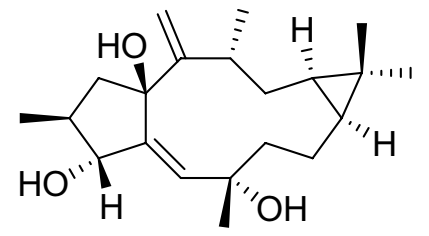

15-deoxy-1 $\beta$-hydroxy-4(4E)jatrogrossidentadione, 123

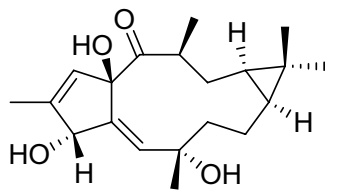

Unsaturated ring A of 15-deoxy-1 $\beta$-hydroxy4(4E)-jatrogrossidentadione, 124 
Table 3 (continued)

\begin{tabular}{|c|c|c|c|c|c|c|}
\hline $\begin{array}{l}\text { Natural product } \\
\text { source }\end{array}$ & Chemical structure & $\begin{array}{l}\text { Class of natural } \\
\text { product }\end{array}$ & $\mathrm{IC}_{50} / \mu \mathrm{g} / \mathrm{mL}$ & $\begin{array}{l}\text { Organism } \\
\text { tested }\end{array}$ & Toxicology & References \\
\hline Psidium Guajava & & Triterpene & 1.01 & $\begin{array}{l}\text { L. infantum } \\
\text { (Axenic Amas- } \\
\text { tigotes) }\end{array}$ & $\begin{array}{c}\text { At conc. }=12.2 \mu \mathrm{M} \text { in } \\
\text { mouse macrophage } \\
\text { cell lines J774A.1 }\end{array}$ & [133] \\
\hline & & & & & $\begin{array}{l}\text { At conc. }=20.8 \mu \mathrm{M} \\
\text { against same cell } \\
\text { lines }\end{array}$ & \\
\hline
\end{tabular}

Corosolic acid, 125

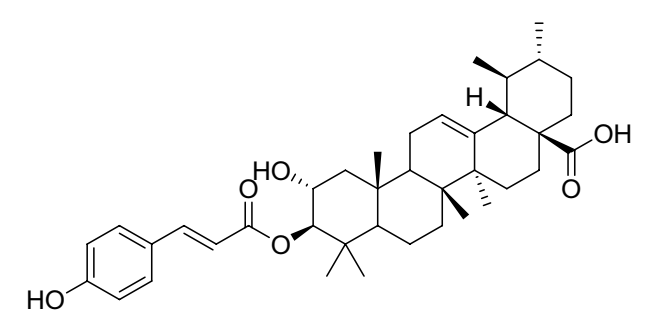

Triterpene $\quad 1.32$

Jacoumaric acid, 126

Cystoseira baccato<smiles>COc1cc(C)c2c(c1)C(=O)C[C@@](C)(CC1=C(CC(C)(C)O)C(=O)[C@@]3(C)CCC[C@]3(C)C1)O2</smiles>

$\begin{array}{ccc}\text { Diterpenoids } 20.4 & \begin{array}{l}\text { L. infantum } \\ \text { (promastig- } \\ \text { otes) }\end{array} & \begin{array}{l}\text { Non-toxic up to } \\ 126.6\end{array} \\ & & \end{array}$

Tetraprenyltoluquinone, 127<smiles>COc1cc(C)c2c(c1)C(O)C[C@@](C)(CC1=C(CC(C)(C)O)C(=O)[C@@]3(C)CCC[C@]3(C)C1)O2</smiles>

Tetraprenyltoluquinol, 128 
Table 3 (continued)

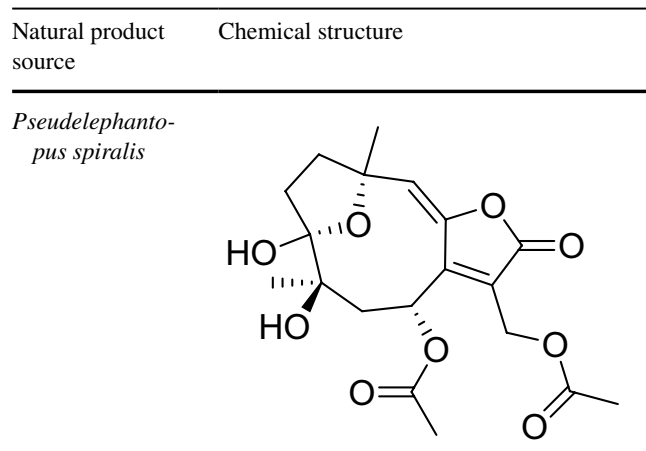

Diacetylpiptocarphol, 129

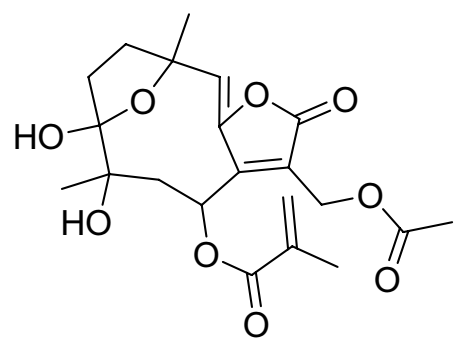

Piptocarphin A, 130

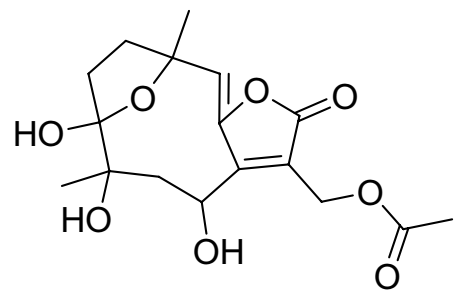

Piptocarphins D, 131

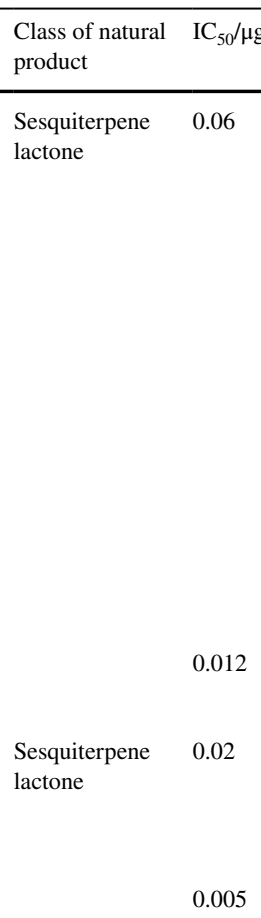

(0)

Sesquiterpene $\quad 0.244$

lactone

0.048
References

tested

L. infantum

(promastig-

otes)

L. infantum

(amastigotes)

L. infantum

(promastig-

otes)

L. infantum

(promastig-

otes)

L. infantum

(amastigotes)

$1.47 \pm 0.08$

$0.97 \pm 0.07$

$5.57 \pm 1.9$

[135] 
Table 3 (continued)

\begin{tabular}{|c|c|c|c|c|c|c|}
\hline $\begin{array}{l}\text { Natural product } \\
\text { source }\end{array}$ & Chemical structure & $\begin{array}{l}\text { Class of natural } \\
\text { product }\end{array}$ & $\mathrm{IC}_{50} / \mu \mathrm{g} / \mathrm{mL}$ & $\begin{array}{l}\text { Organism } \\
\text { tested }\end{array}$ & Toxicology & References \\
\hline $\begin{array}{l}\text { Nectria pseudotri- } \\
\quad \text { chia }\end{array}$ & $\mathrm{HO}^{-}$ & $\begin{array}{l}\text { Sesquiterpene } \\
\text { Lactone }\end{array}$ & $\begin{array}{l}0.092 \\
0.023\end{array}$ & $\begin{array}{l}\text { L. infantum } \\
\text { (promastig- } \\
\quad \text { otes) } \\
\text { L. infantum } \\
\text { (amastigotes) }\end{array}$ & $3.17 \pm 1.0$ & [136] \\
\hline
\end{tabular}

[(2Z)-8,10,11-trihydroxy-1,10-dimethyl-5-oxo4,14-dioxatricyclo[9.2.1.03,7]tetradeca-2,6dien-6-yl]methyl acetate, 132<smiles>CC(=O)OC1(CO)CCC(C(C)C)C2C=C(C(=O)O)COC(=O)C21</smiles>
L. braziliensis
(amastigotes)
Highly selective to parasites compared to VERO cells and THP-1 (a human leukaemia monocytic cell line). All $>200 \mu \mathrm{M}$.

10-acetyl trichoderonic acid A, 133<smiles>CC(=O)OCCCCC/C=C(/C(=O)O)[C@H](C)C(=O)O</smiles>

Monoterpene $\quad 0.104$

6'-acetoxy-piliformic acid, 134<smiles>C=CCCC/C=C(/C(=O)O)[C@H](C)C(=O)O</smiles>

5',6'-dehydropiliformic acid, 135<smiles>CCCCC/C=C(\C(=O)O)[C@H](C)C(=O)O</smiles>

Monoterpene $\quad 0.37$ 
Table 3 (continued)

\begin{tabular}{llllll}
\hline $\begin{array}{l}\text { Natural product } \\
\text { source }\end{array}$ & Chemical structure & $\begin{array}{l}\text { Class of natural } \\
\text { product }\end{array}$ & $\mathrm{IC}_{50} / \mu \mathrm{g} / \mathrm{mL}$ & $\begin{array}{l}\text { Organism } \\
\text { tested }\end{array}$ & $\begin{array}{c}\text { Toxicology } \\
\text { ensis } \\
\text { (promastig- } \\
\text { otes) }\end{array}$ \\
\hline Croton echioides & Diterpenoid & 0.11 & N.T & [137]
\end{tabular}

Methyl-15,16-Epoxy-3,13(16),14-

Neo-Clerodatrien-17,18-Dicarboxylate, 137<smiles>COC(=O)C1=CCC[C@]2(C)[C@H](CCc3ccco3)[C@H](C(=O)OC)CC[C@]12C</smiles>

\section{Nasimalun B, 138}<smiles>COC(=O)C1=CCC[C@@H]2[C@]1(C)CC[C@@H](C)[C@]2(C)CCc1ccoc1</smiles>

Hardwickiic acid methyl ester, 139

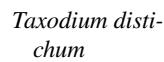<smiles>COCC(=O)[C@H]1C(C)(C)CCC[C@]1(C)C1=C(O)C(=O)C(C(C)C)=CC1=O</smiles>

Taxotrione, 140

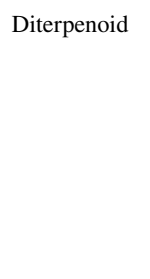

2.5
L. donovani (promastig-

otes)

High toxicity against HT-29 colorectal carcinoma cells

0.52 ensis 
Table 3 (continued)

\begin{tabular}{|c|c|c|c|c|c|c|}
\hline $\begin{array}{l}\text { Natural product } \\
\text { source }\end{array}$ & Chemical structure & $\begin{array}{l}\text { Class of natural } \\
\text { product }\end{array}$ & $\mathrm{IC}_{50} / \mu \mathrm{g} / \mathrm{mL}$ & $\begin{array}{l}\text { Organism } \\
\text { tested }\end{array}$ & Toxicology & References \\
\hline Lippia sidoides & & Monoterpene & 23.9 & $\begin{array}{l}\text { L. amazon- } \\
\text { ensis } \\
\text { (Promastig- } \\
\text { otes) }\end{array}$ & $\begin{array}{l}36.5 \mu \mathrm{M} \\
>100 \mu \mathrm{M} \\
63.6 \mu \mathrm{M}\end{array}$ & [139] \\
\hline
\end{tabular}

Thymol, 141<smiles>CC(=O)c1c(C)ccc(C(C)C)c1O</smiles>

Acetyl-Thymol, 142<smiles>Cc1ccc(C(C)C)c(O)c1C(=O)c1ccccc1</smiles>

\section{Benzoyl-Thymol, 143}

Trixis antimenorrhoea<smiles></smiles>

$\begin{array}{ccl} & 0.3 & \begin{array}{l}\text { L. amazon- } \\ \text { ensis } \\ \text { (promastigote) }\end{array} \\ \text { Sesquiterpene } & 0.96 & \begin{array}{l}\text { L. } \text { brazilensis } \\ \text { (promastigote) }\end{array}\end{array}$
(promastigote)

Bifurcaria bifurc-<smiles>CC(C)=C[C@@H](O)C/C(C)=C/CC[C@](C)(O)CCC/C(C)=C/CO</smiles> 
Table 3 (continued)

\begin{tabular}{|c|c|c|c|c|c|c|}
\hline $\begin{array}{l}\text { Natural product } \\
\text { source }\end{array}$ & Chemical structure & $\begin{array}{l}\text { Class of natural } \\
\text { product }\end{array}$ & $\mathrm{IC}_{50} / \mu \mathrm{g} / \mathrm{mL}$ & $\begin{array}{l}\text { Organism } \\
\text { tested }\end{array}$ & Toxicology & References \\
\hline $\begin{array}{l}\text { Dictyota spiralis } \\
\text { (Marine) }\end{array}$ & & Diterpene & 15.47 & $\begin{array}{l}\text { L. amazon- } \\
\quad \text { ensis } \\
\text { (promastigote) } \\
\text { L. amazon- } \\
\quad \text { ensis } \\
\text { (promastigote) }\end{array}$ & $\begin{array}{l}23.4 \\
69\end{array}$ & [141] \\
\hline
\end{tabular}

spiralyde A, 146

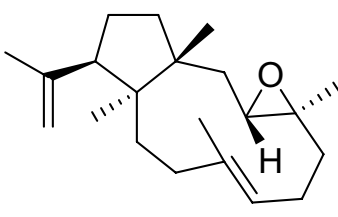

Diterpene $\quad 36.81$

3,4-epoxy-7,18-dolabelladiene, 147

Stypopodium

zonale<smiles>COc1cc(C)c(O)c(C[C@H]2[C@@H](C)CC[C@H]3[C@H](CCC(=O)O)C(=C(C)C)CC[C@H]3[C@H]2C)c1</smiles>

Diterpene 9
L. amazon- $\quad 8.4 \mu \mathrm{M}$
ensis
(amastigotes)

[142]

(Marine)

Atomaric acid, 148 
Table 3 (continued)

\begin{tabular}{|c|c|c|c|c|c|c|}
\hline $\begin{array}{l}\text { Natural product } \\
\text { source }\end{array}$ & Chemical structure & $\begin{array}{l}\text { Class of natural } \\
\text { product }\end{array}$ & $\mathrm{IC}_{50} / \mu \mathrm{g} / \mathrm{mL}$ & $\begin{array}{l}\text { Organism } \\
\text { tested }\end{array}$ & Toxicology & References \\
\hline $\begin{array}{l}\text { Plumarella deli- } \\
\text { catissima } \\
\text { (Marine) }\end{array}$ & & Diterpene & 0.025 & $\begin{array}{l}\text { L. donovani } \\
\text { (amastigotes) }\end{array}$ & $\begin{array}{l}\text { Cytotoxicity potential } \\
\text { against human lung } \\
\text { carcinoma, cells } \\
\text { exhibited low toxic } \\
\text { potentials which } \\
\text { were } \\
>50\end{array}$ & \\
\hline
\end{tabular}

Keikipukalide B, 149<smiles>C=C(C)[C@@H]1CC=C2C(OC(C)=O)[C@H](OC(C)=O)[C@@]3(C)C[C@]4(C)O[C@H]4c4cc(C=O)c(o4)OC23O1</smiles>

Diterpene $\quad 0.026 \quad>50$

Keikipukalide C, 150

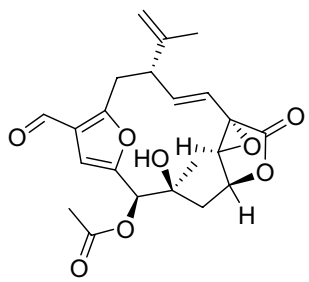

$\begin{array}{lll}\text { Diterpene } & 0.034 & >50\end{array}$

Keikipukalide D, 151

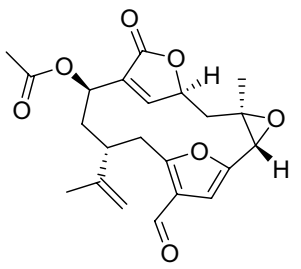

$\begin{array}{lll}\text { Diterpene } & 0.022 & >50\end{array}$

Keikipukalide

E, 152<smiles>C=C(C)[C@H]1CCC2=C[C@@H](C[C@@H]3O[C@H]3[C@H]3C=CC(C=O)=C3C2)OC1=O</smiles>

Pukalide aldehyde, 153

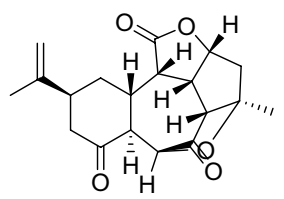


Table 3 (continued)

\begin{tabular}{|c|c|c|c|c|c|c|}
\hline $\begin{array}{l}\text { Natural product } \\
\text { source }\end{array}$ & Chemical structure & $\begin{array}{l}\text { Class of natural } \\
\text { product }\end{array}$ & $\mathrm{IC}_{50} / \mu \mathrm{g} / \mathrm{mL}$ & $\begin{array}{l}\text { Organism } \\
\text { tested }\end{array}$ & Toxicology & References \\
\hline $\begin{array}{l}\text { Laurencia viridis } \\
\text { (Marine) }\end{array}$ & $\mathrm{HC}$ & Diterpene & $\begin{array}{l}8.36 \\
28.26\end{array}$ & $\begin{array}{l}\text { L. amazonen- } \\
\text { sis (Promas- } \\
\text { tigote) } \\
\text { L. donovani } \\
\text { (promastig- } \\
\text { otes) }\end{array}$ & 0.22 & [143] \\
\hline
\end{tabular}

Dehydrothyrsiferol, 155

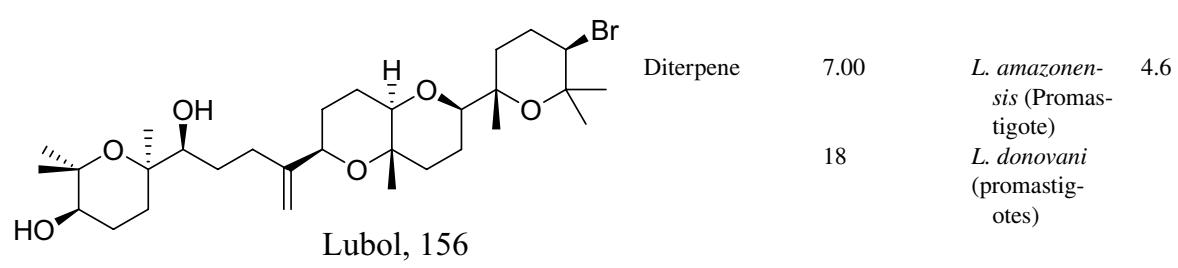<smiles>C=C(CC[C@@H](O)[C@]1(C)CC[C@@](O)(C(C)(C)O)O1)[C@H]1CC[C@H]2O[C@H]([C@]3(C)CC[C@@H](Br)C(C)(C)O3)CC[C@]2(C)O1</smiles>

22-hydroxydehydrothyrsiferol, 157

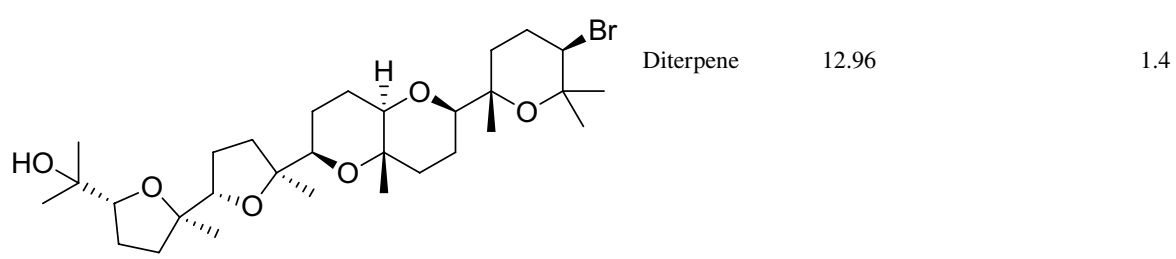

Saiyacenols A, 158<smiles>CC(C)(O)[C@@H]1CCC([C@@]2(C)CCC([C@@H]3CC[C@H]4O[C@@H]([C@]5(C)CC[C@H](Br)C(C)(C)O5)CC[C@@]4(C)O3)O2)O1</smiles>

Saiyacenols B, 159 


\begin{tabular}{|c|c|c|c|c|c|c|}
\hline $\begin{array}{l}\text { Natural product } \\
\text { source }\end{array}$ & Chemical structure & $\begin{array}{l}\text { Class of natural } \\
\text { product }\end{array}$ & $\mathrm{IC}_{50} / \mu \mathrm{g} / \mathrm{mL}$ & $\begin{array}{l}\text { Organism } \\
\text { tested }\end{array}$ & Toxicology & References \\
\hline $\begin{array}{l}\text { Dysidea avara } \\
\text { (Marine) }\end{array}$ & & Sesquiterpene & $\begin{array}{l}28.21 \\
20.28 \\
7.64\end{array}$ & $\begin{array}{l}\text { L. infantum } \\
\text { (Promastig- } \\
\quad \text { otes) } \\
\text { L. tropica } \\
\text { (Promastig- } \\
\quad \text { otes) } \\
\text { L. infantum } \\
\text { (Amastigote) }\end{array}$ & $\begin{array}{l}\text { Low toxicity against } \\
\text { human microvascu- } \\
\text { lar endothelial cells } \\
\text { and (human acute } \\
\text { monocytic leukemia } \\
\text { cells with CC50 } \\
62.19 \text { and }>100 \\
\text { respectively. }\end{array}$ & [144] \\
\hline
\end{tabular}

Avarone, 160<smiles></smiles>

$\begin{array}{cccc} & 7.42 & \begin{array}{l}\text { L. infantum } \\ \text { (Promastig- } \\ \text { otes) }\end{array} & 36.8 \\ \text { Sesquiterpene } & 7.08 & \begin{array}{l}\text { L. tropica } \\ \text { (Promastig- } \\ \text { otes) }\end{array} & 31.75 \\ & 3.19 & \begin{array}{l}\text { L. infantum } \\ \text { (Amastigote) }\end{array} & \end{array}$

Avarol, 161

membered ring. Ergosterol, the main sterol in Leishmania parasite constitute a major component of the cell membrane and mitochondrion of the parasite which when inhibited leads to parasite death. 164 extracted from Trametes versicolor mimics Leishmania ergosterol due to similarities in core structure but a break in oxygen-oxygen bond in ergosterol peroxide unleashes oxidation on lipids, proteins and nucleic acids of the parasite by free radical reaction leading to serious toxicity to the Leishmania parasite [145]. Apart from the biological formation of bridge peroxides, the deleterious effects of other lanostane type steroids on membrane state and integrity causing parasite death has been reported [146, 147]. Also, anti-infective studies of Sassafras albidum and its bioactivity guided fractionation reported a sterol and fatty alcohol, 162 and 163 respectively [36] as promising antileishmanial compounds. 162 which differs from cholesterol at $\mathrm{C} 24$ position is believed to kill the parasite via an apoptosis mechanism involving DNA fragmentation, inhibition of inflammation cytokines and the activation of caspases [148, 149]. Evaluating the suicidal action of active isolates from Pentalinon andrieuxii, 181 induced changes in immune responses particularly via necrosis and apoptosis characterized by increase in IL 2 and IFN- $\gamma$ which insinuates the control of pro-inflammatory cytokines by anti-inflammatory counterparts [150]. 182 halted the process of electron transport and ATP generation in the mitochondria [151]. In addition, plasma membrane alterations with the administration of the other isolates depicts a sterol metabolism inhibition as a contributing factor to parasite death [151].

\section{Conclusion}

Though humans and natural products did not co-evolve, chemical prototypes from natural origins have numerous targets in both human and animal diseases. Their structural diversity, large hemical space and safety are intriguing characteristics that makes them very attractive. Diverse biomolecular functions including anti-leishmanial potentials are possessed by various plant families including Fabaceae, Annonaceae, Euphorbiaceae, Rutaceae, Myrsinaceae, Liliaceae, Araliaceae and Simaroubaceae, as well as endophytes genera Alternaria, Arthrinium, Penicillium, Cochloibus, Fusarium, Colletotrichum, and Gibberella, and marine natural product possess. 
Table 4 Various classes of steroids, fatty alcohol, lignan, and butanolide with their $\mathrm{IC}_{50}$ exhibiting antileishmanial properties

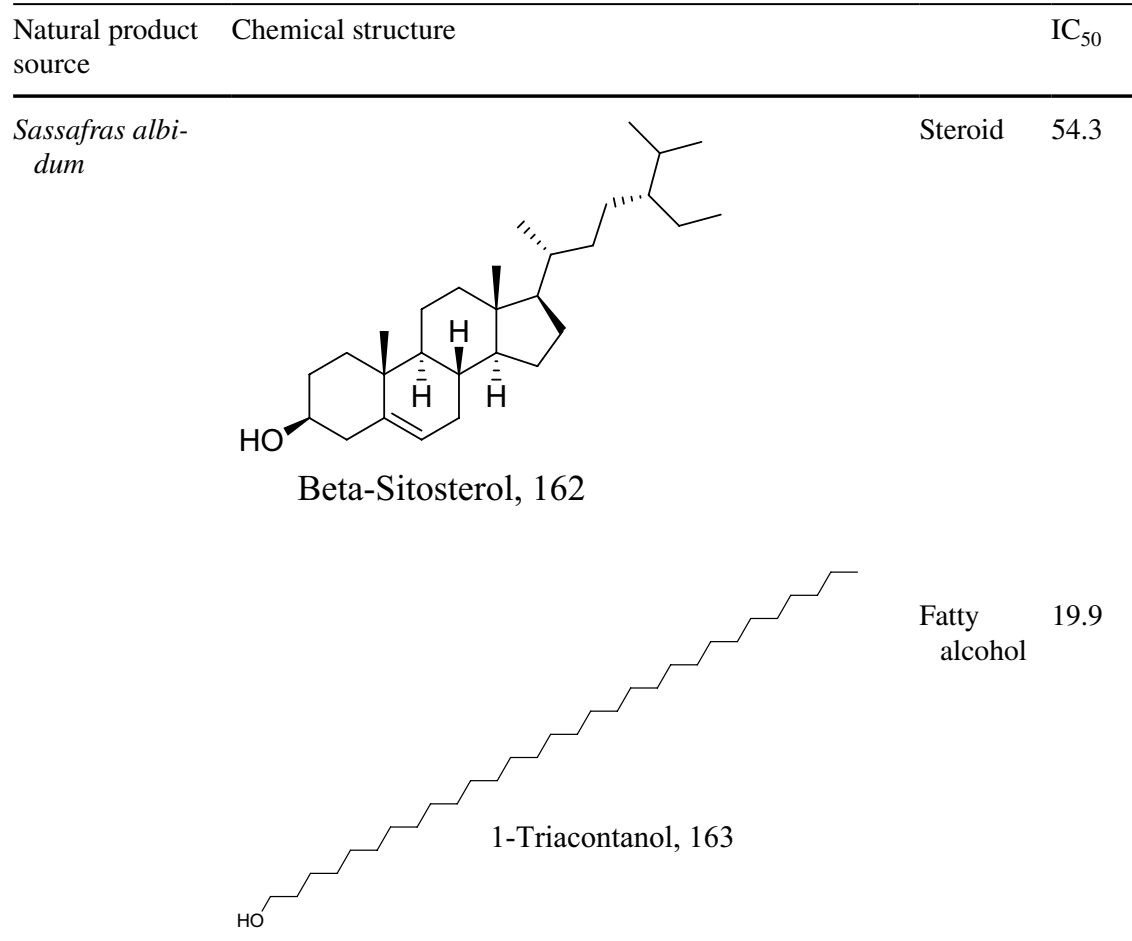

Organism
tested
tested

$\begin{array}{ll}\begin{array}{l}\text { L. amazon- } \\ \text { ensis }\end{array} & \begin{array}{l}\text { Nontoxic against } \\ \text { (PALB/c mouse }\end{array} \\ \begin{array}{l}\text { Promastig- } \\ \text { ote) }\end{array} & \begin{array}{l}\text { macrophages } \\ \text { up to }\end{array} \\ & 182\end{array}$

[36]

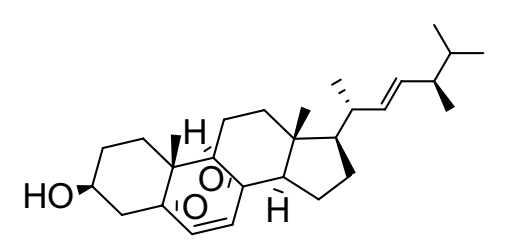
L. amazon- Toxicity profile
$\begin{array}{ll}\text { ensis } & \text { against } \\ \text { Amastigote) } & \text { peritoneal }\end{array}$

Steroid macrophages

color

\section{Ergosterol peroxide, 164}<smiles>CC(C)=CCC[C@H](C(=O)O)C1CC[C@]2(C)C3=C(CC[C@]12C)[C@@]1(C)CCC(O)C(C)(C)[C@@]1(C)CC3</smiles>

Trametenolic acid B, 165 
Table 4 (continued)

$\begin{aligned} & \text { Natural product } \\ & \text { source }\end{aligned}$
$\begin{aligned} & \text { Aspergillus ter- } \\ & \text { reus }\end{aligned}$

(22E,24R)-stigmasta-5,7,22-trien-3-ol, 166

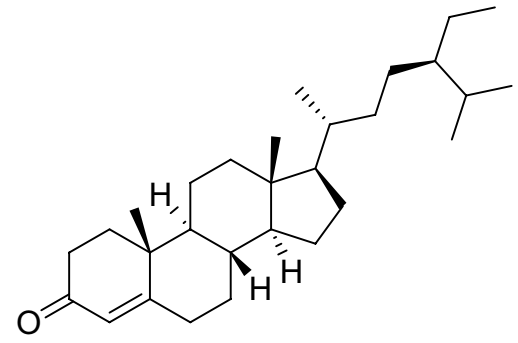

Stigmast-4-ene-3-one, 167<smiles>CCC(/C=C/[C@H](C)[C@H]1CCC2=C3C=CC4=CC(=O)CC[C@]4(C)[C@H]3CC[C@]21C)C(C)C</smiles>

Stigmasta-4,6,8(14),22-tetraen-3-one, 168<smiles>CCOC1C(=O)OC(Cc2ccc(O)cc2)(C(=O)OC)C1c1ccc(O)cc1</smiles>

Terrenolide S, 169
Steroid $\quad 15.3$

Steroid $\quad 54.3$

Bute- $\quad 7.27$

nolide 
Table 4 (continued)

\begin{tabular}{|c|c|c|c|c|c|c|}
\hline $\begin{array}{l}\text { Natural product } \\
\text { source }\end{array}$ & Chemical structure & & $\mathrm{IC}_{50}$ & $\begin{array}{l}\text { Organism } \\
\text { tested }\end{array}$ & Toxicity & References \\
\hline $\begin{array}{l}\text { Solanum sisym- } \\
\text { briifolium }\end{array}$ & & Steroid & $\begin{array}{l}6.60 \\
3.10\end{array}$ & $\begin{array}{l}\text { L. amazon- } \\
\text { ensis } \\
\text { L. brazilen- } \\
\text { sis }\end{array}$ & N.T & {$[127]$} \\
\hline
\end{tabular}

Cilistol A, 170<smiles>C[C@H](C1C[C@]2(C)OC2(C)C(O)O1)[C@]1(O)CC[C@H]2[C@@H]3CC(O)C4(O)CC=CC(=O)[C@]4(C)[C@H]3CC[C@]21C</smiles>

$\begin{array}{lll}> & \text { L. amazon- } & \text { N. T }\end{array}$

Steroid ensis

$59.8 \quad$ L. brazilen-

sis

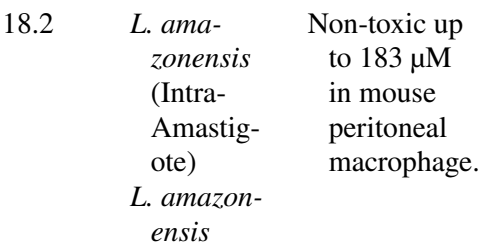<smiles>COc1ccc(C(=O)O)cc1CC=C(C)CCC=C(C)C</smiles>

3-(3,7-dimethyl-2,6-octadienyl)4-methoxybenzoic acid, 173
Paecilomyces

sp.

(Marine)<smiles>O=C1O[C@H](Cc2ccccc2)C[C@H]1O</smiles>

Harzialactone, 172

ensis 
Table 4 (continued)

Natural product Chemical structure

$\mathrm{IC}_{50}$

Organism

Toxicity

References

source

Steroid

201

L. infantum
(Amastigote)

Low toxicity

[155]

siaca

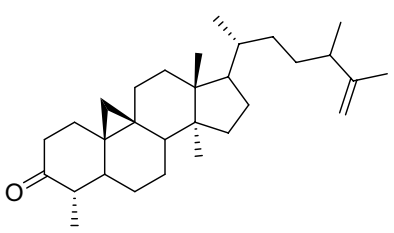

31-Norcyclolaudenone, 174<smiles>C=C(CC[C@@H](C)C1CC[C@H]2[C@H]3CC[C@H]4[C@@H](C)C(=O)CC[C@]45C[C@@]35CC[C@@]12C)C(C)C</smiles>

Cycloeucalenone, 175

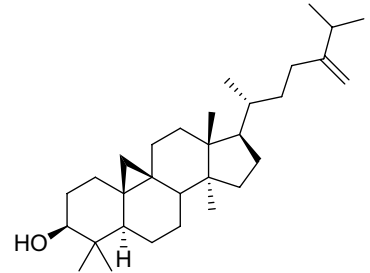

24-Methylene-cycloartanol, 176

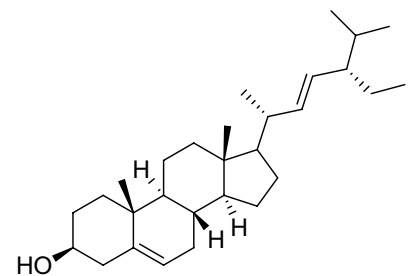

Stigmasterol, 177

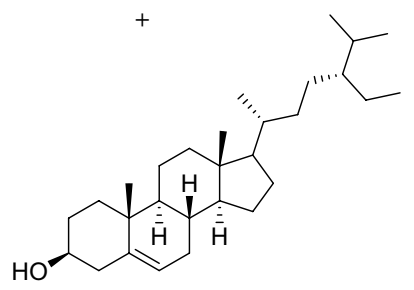

Sitosterol, 178
Steroid 185

$569 \mu \mathrm{M}$ mammalian

raw cell lines

$462 \mu \mathrm{M}$

Steroid 185

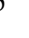

Steroid

127

$1147 \mu \mathrm{M}$

Steroid

98.5

$150 \mu \mathrm{M}$ 
Table 4 (continued)

$\begin{aligned} & \text { Natural product } \\ & \text { source }\end{aligned}$
$\begin{gathered}\text { Pentalinon } \\ \text { andrieuxi }\end{gathered}$

Cholestra-4,20,24-trien-3-one, 179<smiles>CC(=O)C1=CC[C@H]2[C@@H]3CCC4=CC(=O)CC[C@]4(C)[C@H]3C[C@H](O)[C@]12C</smiles>

\section{6,7-Dihydroneridienone, 180}

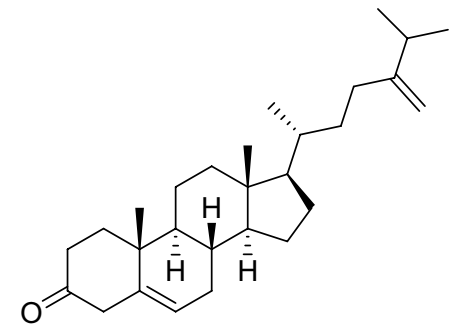

$\begin{array}{rr}\text { Steroid } & 0.06 \\ & 0.009\end{array}$

24-Methylcholesta-4,24(28)-dien-3-one, 181 
Table 4 (continued)

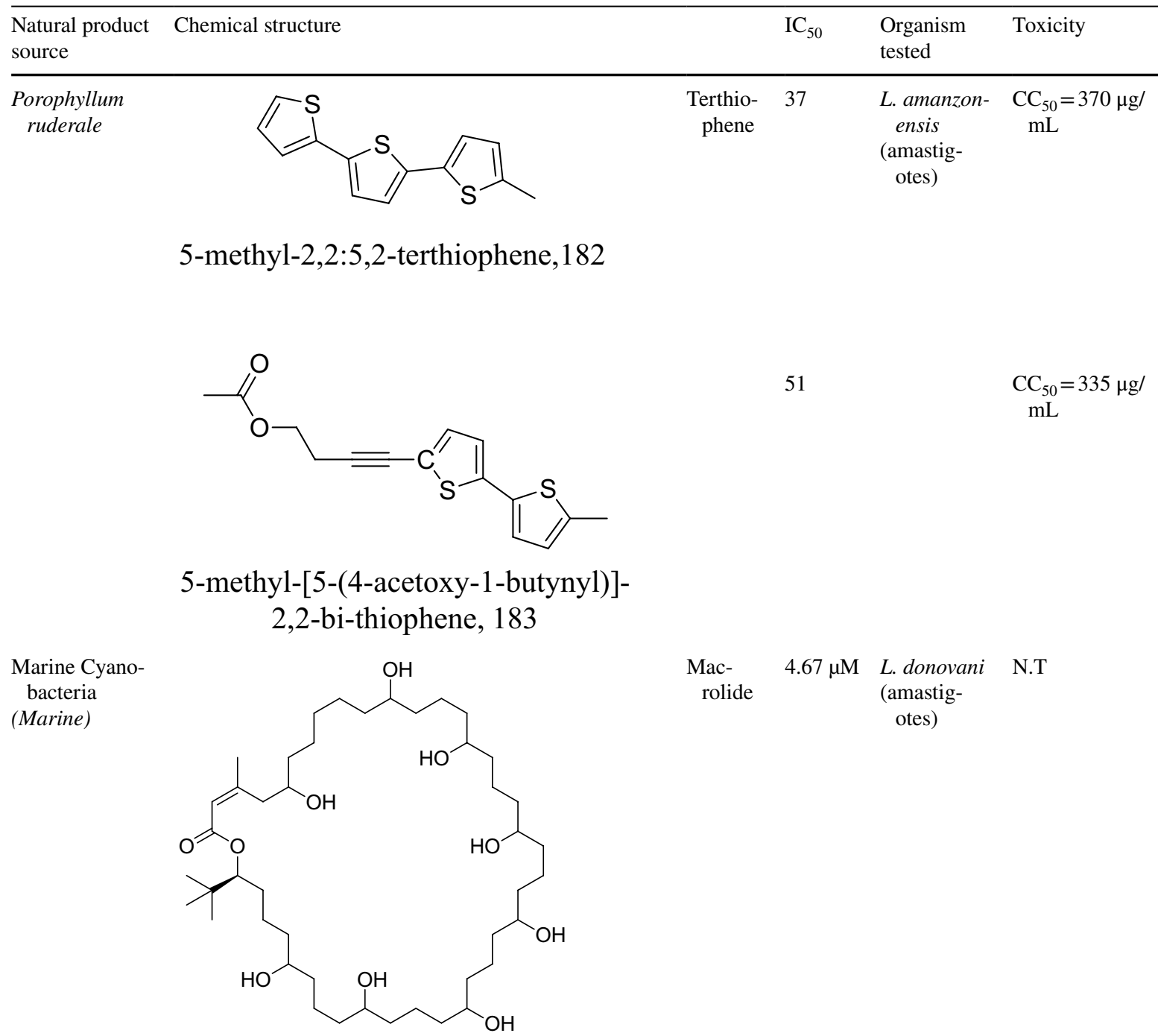

Palstimolide, 184

Management of leishmaniasis is plagued with systemic toxicity, high cost of existing drugs, lengthy treatment periods, drug resistance and parasite diversity. Different classes of natural products such as alkaloids, terpenes, terpenoids, and phenolics are examples of compounds evaluated towards the treatment of leishmaniasis. They exert their antileishmanial activities through calcium channel inhibitors, immunomodulatory through the enhancement of NO in macrophages, alterations in organelle membranes of the endoplasmic reticulum, respiration incapacitation and apoptosis. Other antileishmanial related mechanisms include cell membrane disruption via sterol biosynthesis inhibition, reactive oxygen species (ROS) generation, iron chelation, arginase inhibition, topoisomerase II intercalation, suppressing NF- $\kappa \mathrm{B}$ expression and other pro-inflammatory, and trypanothione reductase inhibition.

Author contributions POS, RKA and SKK initiated the work, POS wrote the first draft supervised by SKK and POS All the authors contributed to the writing of the review, read and accepted the final draft article.

\section{Declarations}

Conflict of interest The authors declare no conflict of interest.

Open Access This article is licensed under a Creative Commons Attribution 4.0 International License, which permits use, sharing, 
adaptation, distribution and reproduction in any medium or format, as long as you give appropriate credit to the original author(s) and the source, provide a link to the Creative Commons licence, and indicate if changes were made. The images or other third party material in this article are included in the article's Creative Commons licence, unless indicated otherwise in a credit line to the material. If material is not included in the article's Creative Commons licence and your intended use is not permitted by statutory regulation or exceeds the permitted use, you will need to obtain permission directly from the copyright holder. To view a copy of this licence, visit http://creativecommons. org/licenses/by/4.0/.

\section{References}

1. T.S. Tiuman, A.O. Santos, T. Ueda-Nakamura, B.P.D. Filho, C.V. Nakamura, Int. J. Infect. Dis. 15, (2011)

2. WHO (2020). https://www.Who.Int/News-Room/Fact-Sheets/ Detail/Leishmaniasis

3. B. Pérez-Cabezas, P. Cecílio, T.B. Gaspar, F. Gärtner, R. Vasconcellos, A. Cordeiro-da-Silva, Front. Cell. Infect. Microbiol. 9, 30 (2019)

4. PAHO/WHO (2017). https://www.paho.org/hq/dmdocuments.

5. I. Okwor, J. Uzonna, Am. J. Trop. Med. Hyg. 94, 489 (2016)

6. R. Arenas, E. Torres-Guerrero, M. R. Quintanilla-Cedillo, and J. Ruiz-Esmenjaud, F1000Research 6, (2017).

7. N. Tiwari, M. R. Gedda, V. K. Tiwari, S. P. Singh, and R. K. Singh, Mini-Reviews Med. Chem. 18, (2018).

8. K. Jain, N.K. Jain, J. Immunol. Methods 422, 1 (2015)

9. S. Emami, P. Tavangar, M. Keighobadi, Eur. J. Med. Chem. 135, 241 (2017)

10. N. Dunning and S. Ali, Bioscience Horizons 2, (2009)

11. S. Srivastava, P. Shankar, J. Mishra, and S. Singh, Parasites and Vectors 9, (2016).

12. P. L. Olliaro, P. J. Guerin, S. Gerstl, A. A. Haaskjold, J. A. Rottingen, and S. Sundar, Lancet Infect. Dis. 5, 763 (2005).

13. P.L. Olliaro, P.J. Guerin, S. Gerstl, A.A. Haaskjold, J.A. Rottingen, S. Sundar, Lancet Infect. Dis. 5, 763 (2005)

14. J. N. Rugani, C. M. F. Gontijo, F. Frézard, R. P. Soares, and R. L. Do Monte-Neto, Mem. Inst. Oswaldo Cruz 114, (2019)

15. F. Frézard, R. Monte-Neto, P.G. Reis, Biophys. Rev. 6, 119 (2014)

16. G. Mandal, S. Mandal, M. Sharma, K.S. Charret, B. Papadopoulou, H. Bhattacharjee, R. Mukhopadhyay, PLoS Negl. Trop. Dis. 9, (2015)

17. S. Sundar, J. Chakravarty, Expert Opin. Pharmacother. 16, 237 (2015)

18. A.C.O. Souza, A.C. Amaral, Front. Microbiol. 8, 336 (2017)

19. A.S. Nagle, S. Khare, A.B. Kumar, F. Supek, A. Buchynskyy, C.J.N. Mathison, N.K. Chennamaneni, N. Pendem, F.S. Buckner, M.H. Gelb, V. Molteni, Chem. Rev. 114, 11305 (2014)

20. A. Tamiru, B. Tigabu, S. Yifru, E. Diro, A. Hailu, B.M.C. Infect, Dis. 16, 548 (2016)

21. J.R. Luque-Ortega, L. Rivas, Antimicrob. Agents Chemother. 51, 1327 (2007)

22. J. Pijpers, M. L. Den Boer, D. R. Essink, and K. Ritmeijer, PLoS Negl. Trop. Dis. 13, 1 (2019).

23. S. Sundar, J. Chakravarty, Expert Opin. Investig. Drugs 17, 787 (2008)

24. V. Wiwanitkit, Ther. Clin. Risk Manag. 8, 323 (2012)

25. W. Khan, R. Kumar, S. Singh, S.K. Arora, N. Kumar, Drug Test. Anal. 5, 468 (2013)

26. P.M. Loiseau, S. Cojean, J. Schrével, J. Parasite, La Société Française Parasitol. 18, 115 (2011)
27. T.J. Gintjee, M.A. Donnelley, G.R. Thompson, J. Fungi 6, 28 (2020)

28. S.T. De Macedo-Silva, J.A. Urbina, W. De Souza, J.C.F. Rodrigues, PLoS ONE 8, (2013)

29. B.B. Mishra, V.K. Tiwari, Eur. J. Med. Chem. 46, 4769 (2011)

30. E. Patridge, P. Gareiss, M.S. Kinch, D. Hoyer, Drug Discov. Today 21, 204 (2016)

31. I. V. Ogungbe, M. Singh, and W. N. Setzer, Antileishmanial Natural Products from Plants, 1st ed. (Elsevier B.V., 2012)

32. D.B. Santana, R.C. da Costa, R.M. Araújo, J.E. de Paula, E.R. Silveira, R. Braz-Filho, L.S. Espindola, Brazilian. J. Pharmacogn. 25, 401 (2015)

33. C.V. Simoben, F. Ntie-Kang, S.H. Akone, W. Sippl, Nat. Products Bioprospect. 8, 151 (2018)

34. N. Ullah, A. Nadhman, S. Siddiq, S. Mehwish, A. Islam, L. Jafri, M. Hamayun, Phyther. Res. 30, 1905 (2016)

35. J. Búfalo, C.L. Cantrell, M.R. Jacob, K.K. Schrader, B.L. Tekwani, T.S. Kustova, A. Ali, C.S.F. Boaro, Planta Med. 82, 131 (2015)

36. D. Pulivarthi, K.M. Steinberg, L. Monzote, A. Piñón, W.N. Setzer, Nat. Prod. Commun. 10, 1229 (2015)

37. L. Scotti, H. Ishiki, F.J.B. Mendonca, M.S. Silva, M.T. Scotti, Mini-Reviews. Med. Chem. 15, 253 (2015)

38. R. Sen, M. Chatterjee, Phytomedicine 18, 1056 (2011)

39. S.K. Venkatesan, P. Saudagar, A.K. Shukla, V.K. Dubey, Interdiscip. Sci. Comput Life Sci. 3, 217 (2011)

40. R.S. Gabriel, A.C.F. Amaral, I.C. Lima, J.D. Cruz, A.R. Garcia, H.A.S. Souza, C.M. Adade, A.B. Vermelho, C.S. Alviano, D.S. Alviano, I.A. Rodrigues, Rev. Bras. Farmacogn. 29, 755 (2019)

41. B.B. Cota, L.H. Rosa, R.B. Caligiorne, A.L.T. Rabello, T.M. AlmeidaAlves, C.A. Rosa, C.L. Zani, FEMS Microbiol. Lett. 285, 177 (2008)

42. L.H. Rosa, V.N. Gonçalves, R.B. Caligiorne, T.M.A. Alves, A. Rabello, P.A. Sales, A.J. Romanha, M.E.G. Sobral, C.A. Rosa, C.L. Zani, Brazilian. J. Microbiol. 41, 420 (2010)

43. D. Pech-Puch, M. Pérez-Povedano, O.A. Lenis-Rojas, J. Rodríguez, C. Jiménez, Mar. Drugs 18, 59 (2020)

44. K. Hayibor, S. Kwain, E. Osei, A.P. Nartey, G.M. Tetevi, K.B.A. Owusu, M. Camas, A.S. Camas, K. Kyeremeh, Int. J. Biol. Chem. Sci. 13, 1918 (2019)

45. M. M. Basyoni, Parasitol. United J. 5, (2012).

46. R. M. de Oliveira, S. de A. Melo, T. A. da Penha-Silva, F. Almeida-Souza, and A. L. Abreu-Silva, in Leishmaniases as Re-Emerging Dis. (InTech, 2018)

47. L.R.T. Yamthe, R. Appiah-Opong, P.V.T. Fokou, N. Tsabang, F.F. Boyom, A.K. Nyarko, M.D. Wilson, Mar. Drugs 15, 323 (2017)

48. I. A. Rodrigues, A. M. Mazotto, V. Cardoso, R. L. Alves, A. C. F. Amaral, J. R. D. A. Silva, A. S. Pinheiro, and A. B. Vermelho, Mediators Inflamm. 2015, (2015)

49. R.M.K. Toghueo, Nat. Products Bioprospect. 9, 311 (2019)

50. N. Fatima, S. Muhammad, A. Mumtaz, H. Tariq, I. Shahzadi, M. Said, M. Dawood, Br. J. Pharm. Res. 12, 1 (2016)

51. M. Oliveira, L. Barreira, K.N. Gangadhar, M.J. Rodrigues, T. Santos, J. Varela, L. Custódio, Phytochem. Rev. 15, 663 (2016)

52. E. C. Da Silva, C. D. Rayol, P. L. Medeiros, R. C. B. Q. Figueiredo, M. R. Piuvezan, J. M. Brabosa-Filho, A. Fernandes Marinho, T. G. Silva, G. C. G. Militão, A. P. P. Cassilhas, and P. P. De Andrade, Sci. World J. 2012, (2012)

53. L.R.C. Guimarães, A.P.D. Rodrigues, P.S.B. Marinho, A.H. Muller, G.M.S. Guilhon, L.S. Santos, J.L.M. Do Nascimento, E.O. Silva, Parasitol. Res. 107, 1075 (2010)

54. S. M. M. Amyra Amat Sain, Azimah Amanah, Zuriati Zahari, Roshan Jahn Mohd Salim, Int. J. Pharmacol. Phytochem. Ethnomedicine 3, 1 (2016). 
55. N. Zhu, X. Cao, P. Hao, Y. Zhang, Y. Chen, J. Zhang, J. Li, C. Gao, L. Li, Cell Stress Chaperones 25, 417 (2020)

56. N. Zorić, I. Kosalec, S. Tomić, I. Bobnjarić, M. Jug, T. Vlainić, J. Vlainić, B.M.C. Complement, Altern. Med. 17, 268 (2017)

57. G.M. Tetevi, S. Kwain, T. Mensah, A.S. Camas, M. Camas, A.K. Dofuor, F.A. Azerigyik, E. Oluwabusola, H. Deng, M. Jaspars, K. Kyeremeh, Molbank 2019, M1094 (2019)

58. E. Osei, S. Kwain, G.T. Mawuli, A.K. Anang, K.B.A. Owusu, M. Camas, A.S. Camas, M. Ohashi, C.N. Alexandru-Crivac, H. Deng, M. Jaspars, K. Kyeremeh, Mar. Drugs 17, 9 (2019)

59. S.L. Shyaula, T. Tamang, N. Ghouri, A. Adhikari, S. Marasini, G.B. Bajracharya, M.D. Manandhar, M.I. Choudhary, Nat. Prod. Res. 30, 2590 (2016)

60. M.E. Ferreira, A. Rojas de Arias, G. Yaluff, N.V. de Bilbao, H. Nakayama, S. Torres, A. Schinini, I. Guy, H. Heinzen, A. Fournet, Phytomedicine 17, 375 (2010)

61. C.B. Naman, G. Gupta, S. Varikuti, H. Chai, R.W. Doskotch, A.R. Satoskar, A.D. Kinghorn, J. Nat. Prod. 78, 552 (2015)

62. A. M. Metwaly, M. M. Ghoneim, and A. Musa, Two new antileishmanial diketopiperazine alkaloids from the endophytic fungus Trichosporum Sp (2015)

63. T. Naz, A. Mosaddik, M.M. Rahman, I. Muhammad, M.E. Haque, S.K. Cho, Nat. Prod. Res. 26, 979 (2012)

64. A.D.C. Cunha, T.P.C. Chierrito, G.M.D.C. MacHado, L.L.P. Leon, C.C. Da Silva, J.C. Tanaka, L.M. De Souza, R.A.C. Gonalves, A.J.B. De Oliveira, Phytomedicine 19, 413 (2012)

65. A. Mollataghi, E. Coudiere, A.H.A. Hadi, M.R. Mukhtar, K. Awang, M. Litaudon, A. Ata, Fitoterapia 83, 298 (2012)

66. H. Mahmoudvand, S.A.A. Mousavi, A. Sepahvand, F. Sharififar, B. Ezatpour, F. Gorohi, E.S. Dezaki, S. Jahanbakhsh, ISRN Pharmacol. 2014, 1 (2014)

67. S. Ghosal, A. Deb, P. Mishra, R. Vishwakarma, Planta Med. 78, 906 (2012)

68. I. Orhan, B. Şener, M. Kaiser, R. Brun, D. Tasdemir, Mar. Drugs 8, 47 (2010)

69. L. Cartuche, I. Sifaoui, A. López-Arencibia, C.J. BethencourtEstrella, D.S. Nicolás-Hernández, J. Lorenzo-Morales, J.E. Piñero, A.R. Díaz-Marrero, J.J. Fernández, Biomolecules 10, 1 (2020)

70. Y.S. Rizk, A. Fischer, M.C. de Cunha, P.O. Rodrigues, M.C.S. Marques, M.F.C. de Matos, M.C.T. Kadri, C.A. Carollo, C.C.P. de Arruda, Mem. Inst. Oswaldo Cruz 109, 1050 (2014)

71. P.S. Lage, M.A. Chávez-Fumagalli, J.T. Mesquita, L.M. Mata, S.O.A. Fernandes, V.N. Cardoso, M. Soto, C.A.P. Tavares, J.P.V. Leite, A.G. Tempone, E.A.F. Coelho, Parasitol. Res. 114, 4625 (2015)

72. F. Fonseca-Silva, J.D.F. Inacio, M.M. Canto-Cavalheiro, E.E. Almeida-Amaral, PLoS ONE 6, (2011)

73. S.S. Grecco, T.A. Costa-Silva, G. Jerz, F.S. de Sousa, G.A.A. Conserva, J.T. Mesquita, M.K. Galuppo, A.G. Tempone, B.J. Neves, C.H. Andrade, R.L.O.R. Cunha, M. Uemi, P. Sartorelli, J.H.G. Lago, Phytomedicine 24, 62 (2017)

74. F.S. de Sousa, S.S. Grecco, N. Girola, R.A. Azevedo, C.R. Figueiredo, J.H.G. Lago, Phytochemistry 140, 108 (2017)

75. L.S. Morais, R.G. Dusi, D.P. Demarque, R.L. Silva, L.C. Albernaz, S.N. Bao, C. Merten, L.M.R. Antinarelli, E.S. Coimbra, L.S. Espindola, PLoS ONE 15, 1 (2020)

76. R. A. Falcao, P. L. A. Do Nascimento, S. A. De Souza, T. M. G. Da Silva, A. C. De Queiroz, C. B. B. Da Matta, M. S. A. Moreira, C. A. Camara, and T. M. S. Silva, Evidence-Based Complement. Altern. Med. 2013, (2013)

77. M. Alagawany, M.E.A. El-Hack, M.R. Farag, M. Gopi, K. Karthik, Y.S. Malik, K. Dhama, Anim. Health. Res. Rev. 18, 167 (2017)
78. V.K. Bajpai, M.B. Alam, K.T. Quan, M.K. Ju, R. Majumder, S. Shukla, Y.S. Huh, M.K. Na, S.H. Lee, Y.K. Han, Sci. Rep. 8, 9216 (2018)

79. R.F. Espírito-Santo, C.S. Meira, R. DosSantosCosta, O.P.S. Filho, A.F. Evangelista, G.H.G. Trossini, G.M. Ferreira, E.S. Da Velozo, C.F. Villarreal, M.B.P. Soares, PLoS ONE 12, 0179174 (2017)

80. W. Gao, Q. Li, J. Chen, Z. Wang, C. Hua, Molecules 18, 15613 (2013)

81. V. P. C. Rocha, C. Q. Da Rocha, E. F. Queiroz, L. Marcourt, W. Vilegas, G. B. Grimaldi, P. Furrer, E. Allémann, J. L. Wolfender, and M. B. P. Soares, Molecules 24, (2019)

82. M.M. Radwan, A.S. Wanas, F.R. Fronczek, M.R. Jacob, S.A. Ross, Med. Chem. Res. 24, 3398 (2015)

83. T.A. Da Costa-Silva, S.S. Grecco, F.S. De Sousa, J.H.G. Lago, E.G.A. Martins, C.A. Terrazas, S. Varikuti, K.L. Owens, S.M. Beverley, A.R. Satoskar, A.G. Tempone, J. Nat. Prod. 78, 653 (2015)

84. A. Kaur, R. Singh, C.S. Dey, S.S. Sharma, K.K. Bhutan, I.P. Singh, Indian J. Exp. Biol. 48, 314 (2010)

85. T.A. Da Costa-Silva, G.A.A. Conserva, A.J. Galisteo, A.G. Tempone, J.H.G. Lago, J. Venom. Anim. Toxins Incl. Trop. Dis. 25, 1 (2019)

86. M.C. Vendrametto, A.O. dos Santos, C.V. Nakamura, B.P.D. Filho, D.A.G. Cortez, T. Ueda-Nakamura, Parasitol. Int. 59, 154 (2010)

87. L.A.O. dos Ferreira, M.M. de Oliveira, F.L. Faleiro, D.B. Scariot, J.S. Boeing, J.V. Visentainer, M.B. Romagnolo, C.V. Nakamura, M.C.T. Truiti, Nat. Prod. Res. 32, 2825 (2018)

88. B.B. Mishra, J.K. Gour, N. Kishore, R.K. Singh, V. Tripathi, V.K. Tiwari, Nat. Prod. Res. 27, 480 (2013)

89. F.L. Dutra, M.M. Oliveira, R.S. Santos, W.S. Silva, D.S. Alviano, D.P. Vieira, A.H. Lopes, Acta Trop. 164, 69 (2016)

90. S.M. de Morais, N.S. Vila-Nova, C.M.L. Bevilaqua, F.C. Rondon, C.H. Lobo, A.A.A.N. De Moura, A.D. Sales, A.P.R. Rodrigues, J.R. De Figuereido, C.C. Campello, M.E. Wilson, H.F. De Andrade, Bioorganic. Med. Chem. 22, 6250 (2014)

91. T. Ueda-Nakamura, R.R. Mendonça-Filho, J.A. Morgado-Díaz, P.K. Maza, B.P.D. Filho, D.A.G. Cortez, D.S. Alviano, M.S.S. do Rosa, A.H.C.S. Lopes, C.S. Alviano, C.V. Nakamura, Parasitol. Int. 55, 99 (2006)

92. A.M. do Nascimento, M.G. Soares, F.K.V.S. da Torchelsen, J.A.V. de Araujo, P.S. Lage, M.C. Duarte, P.H.R. Andrade, T.G. Ribeiro, E.A.F. Coelho, A.M. do Nascimento, World J. Microbiol. Biotechnol. 31, 1793 (2015)

93. R. Parise-Filho, K.F.M. Pasqualoto, F.M.M. Magri, A.K. Ferreira, B.A.V.G. Da Silva, M.C.F.C.B. Damião, M.T. Tavares, R.A. Azevedo, A.V.V. Auada, M.C. Polli, C.A. Brandt, Arch. Pharm. (Weinheim). 345, 934 (2012)

94. L.G. Malak, M.A. Ibrahim, D.W. Bishay, A.M. Abdel-Baky, A.M. Moharram, B. Tekwani, S.J. Cutler, S.A. Ross, J. Nat. Prod. 77, 1987 (2014)

95. L.G. Malak, M.A. Ibrahim, A.M. Moharram, P. Pandey, B. Tekwani, R.J. Doerksen, D. Ferreira, S.A. Ross, J. Nat. Prod. 81, $2222(2018)$

96. S. Bashir, M. Alam, A. Adhikari, R.L. Shrestha, S. Yousuf, B. Ahmad, S. Parveen, A. Aman, M.I. Choudhary, Phytochem. Lett. 9, 46 (2014)

97. E.M. Maldonado, E. Salamanca, A. Giménez, G. Saavedra, O. Sterner, Phytochem. Lett. 10, 281 (2014)

98. B. Attioua, L. Lagnika, D. Yeo, C. Antheaume, M. Kaiser, B. Weniger, A. Lobstein, C. Vonthron-Sénécheau, Int. J. Pharm. Sci. Rev. Res. 11, 1 (2011)

99. R.S. Costa, O.P.S. Filho, O.C.S.D. Júnior, J.J. Silva, M. LeHyaric, M.A.V. Santos, E.S. Velozo, Braz. J. Pharmacogn. 28, 551 (2018) 
100. A.O. dos Santos, E.A. Britta, E.M. Bianco, T. Ueda-Nakamura, B.P.D. Filho, R.C. Pereira, C.V. Nakamura, Mar. Drugs 9, 2369 (2011)

101. A. Santos, E. Britta, T. Ueda-Nakamura, B. D. Filho, E. Bianco, V. Teixeira, R. Pereira, and C. Nakamura, Planta Med. 75, (2009)

102. C. Garcia, C.O. Silva, C.M. Monteiro, M. Nicolai, A. Viana, J.M. Andrade, I. Barasoain, T. Stankovic, J. Quintana, I. Hernández, I. González, F. Estévez, A.M. Díaz-Lanza, C.P. Reis, C.A.M. Afonso, M. Pesic, P. Rijo, Future. Med. Chem. 10, 1177 (2018)

103. P. Sitarek, M. Toma, E. Ntungwe, T. Kowalczyk, E. Skała, J. Wieczfinska, T. Śliwiński, P. Rijo, Biomolecules 10, 194 (2020)

104. V.C. Desoti, D. Lazarin-Bidóia, D.B. Sudatti, R.C. Pereira, A. Alonso, T. Ueda-Nakamura, B.P.D. Filho, C.V. Nakamura, S.O. de Silva, Mar. Drugs 10, 1631 (2012)

105. Y. Freile-Pelegrín, D. Tasdemir, Bot. Mar. 62, 211 (2019)

106. A. V. Colares, F. Almeida-Souza, N. N. Taniwaki, C. D. S. F. Souza, J. G. M. Da Costa, K. D. S. Calabrese, and A. L. AbreuSilva, Evidence-Based Complement. Altern. Med. 2013, (2013)

107. S. Hajaji, I. Sifaoui, A. López-Arencibia, M. Reyes-Batlle, I.A. Jiménez, I.L. Bazzocchi, B. Valladares, H. Akkari, J. LorenzoMorales, J.E. Piñero, Parasitol. Res. 117, 2855 (2018)

108. G.S. Lima, D.B. Castro-Pinto, G.C. MacHado, M.A.M. Maciel, A. Echevarria, Phytomedicine 22, 1133 (2015)

109. M. Saleem, Cancer Lett. 285, 109 (2009)

110. Y.L. Liu, S. Lindert, W. Zhu, K. Wang, J.A. McCammon, E. Oldfield, Proc. Natl. Acad. Sci. USA 111, E2530 (2014)

111. Y. Jia, C. Wu, B. Zhang, Y. Zhang, J. Li, Hum. Exp. Toxicol. 38, 227 (2019)

112. K. Becker, S. Schwaiger, B. Waltenberger, D. Fuchs, C. K. Pezzei, H. Schennach, H. Stuppner, and J. M. Gostner, Oxid. Med. Cell. Longev. (2018).

113. D. Slameňová, I. Mašterová, J. Lábaj, E. Horváthová, P. Kubala, J. Jakubíková, L. Wsólová, Basic Clin. Pharmacol. Toxicol. 94 282 (2004)

114. L. Monzote, A. Lackova, K. Staniek, O. Cuesta-Rubio, L. Gille, Parasitology 142, 1239 (2015)

115. L.A. Caldas, M.L. Yoshinaga, M.J.P. Ferreira, J.H.G. Lago, A.B. de Souza, M.D. Laurenti, L.F.D. Passero, P. Sartorelli, Bioorg. Chem. 83, 348 (2019)

116. H. Wu, F.R. Fronczek, C.L. Burandt, J.K. Zjawiony, Planta Med. 77, 749 (2011)

117. Y. Nakagawa, M. Iinuma, N. Matsuura, K. Yi, M. Naoi, T. Nakayama, Y. Nozawa, Y. Akao, J. Pharmacol. Sci. 97, 242 (2005)

118. N. Fakhrudin, B. Waltenberger, M. Cabaravdic, A.G. Atanasov, C. Malainer, D. Schachner, E.H. Heiss, R. Liu, S.M. Noha, A.M. Grzywacz, J. Mihaly-Bison, E.M. Awad, D. Schuster, J.M. Breuss, J.M. Rollinger, V. Bochkov, H. Stuppner, V.M. Dirsch, Br. J. Pharmacol. 171, 1676 (2014)

119. E.S. Yamamoto, B.L.S. Campos, J.A. Jesus, M.D. Laurenti, S.P. Ribeiro, E.G. Kallás, M. Rafael-Fernandes, G. Santos-Gomes, M.S. Silva, D.P. Sessa, J.H.G. Lago, D. Levy, L.F.D. Passero, PLOS ONE 10, (2015)

120. B. Attioua, D. Yeo, L. Lagnika, R. Harisolo, C. Antheaume, B. Weniger, M. Kaiser, A. Lobstein, C. Vonthron-Sénécheau, Pharm. Biol. 50, 801 (2012)

121. F.A. Azerigyik, M. Amoa-Bosompem, T. Tetteh, F. Ayertey, A.N. Antwi, K.B.-A. Owusu, K.K. Dadzie, G.I. Djameh, M. TettehTsifoanya, S. Iwanaga, A.A. Appiah, T. Ohta, T. Uto, Y. Shoyama, N. Ohta, T.M. Gwira, M. Ohashi, European. J. Med. Plants 25, 1 (2018)

122. I.G. Demarchi, M.V. Thomazella, M.S. de Terron, L. Lopes, Z.C. Gazim, D.A.G. Cortez, L. Donatti, S.M.A. Aristides, T.G.V. Silveira, M.V.C. Lonardoni, Exp. Parasitol. 157, 128 (2015)

123. F.L.S. Da MacHado, W. Pacienza-Lima, B. Rossi-Bergmann, L.M.S. De Gestinari, M.T. Fujii, J.C. Paula, S.S. Costa, N.P. Lopes, C.R. Kaiser, A.R. Soares, Planta Med. 77, 733 (2011)
124. C.B.G. Teles, L.S. Moreira, A.D.A.E. Silva, V.A. Facundo, J.P. Zuliani, R.G. Stábeli, I. Silvm, J. Braz. Chem. Soc. 22, 936 (2011)

125. J.A. Crentsil, L.R.T. Yamthe, B.Z. Anibea, E. Broni, S. K. Kwofie, J.K.A. Tetteh, and D. Osei-Safo, Front. Pharmacol. 11, (2020)

126. A. Das, J.J. Jawed, M.C. Das, P. Sandhu, U.C. De, B. Dinda, Y. Akhter, S. Bhattacharjee, Int. J. Antimicrob. Agents 50, 512 (2017)

127. V.C. Filho, C. Meyre-Silva, R. Niero, L.N. Bolda Mariano, F. Gomes Do Nascimento, I. Vicente Farias, V.F. Gazoni, B. Dos Santos Silva, A. Giménez, D. Gutierrez-Yapu, E. Salamanca, and A. Malheiros, Evidence-Based Complement. Altern. Med. 2013, (2013)

128. B.P. da Silva, D.A. Cortez, T.Y. Violin, B.P.D. Filho, C.V. Nakamura, T. Ueda-Nakamura, I.C.P. Ferreira, Parasitol. Int. 59, 643 (2010)

129. S. Singh, U. Sharma, P. Kumar, D. Singh, M. Dobhal, Indian J. Med. Res. 134, 709 (2011)

130. G. Odonne, G. Herbette, V. Eparvier, G. Bourdy, R. Rojas, M. Sauvain, D. Stien, J. Ethnopharmacol. 137, 875 (2011)

131. J.A. Murillo, J.F. Gil, Y.A. Upegui, A.M. Restrepo, S.M. Robledo, W. Quiñones, F. Echeverri, A.S. Martin, H.F. Olivo, G. Escobar, Bioorganic. Med. Chem. 27, 153 (2019)

132. A. Falodun, V. Imieje, O. Erharuyi, A. Joy, P. Langer, M. Jacob, S. Khan, M. Abaldry, M. Hamann, Doc. Head. Asian Pac J Trop Biomed. 4, 374 (2014)

133. C. Phakeovilay, S. Bourgeade-Delmas, P. Perio, A. Valentin, F. Chassagne, E. Deharo, K. Reybier, G. Marti, Molecules 24, 4536 (2019)

134. C. Bruno de Sousa, K.N. Gangadhar, T.R. Morais, G.A.A. Conserva, C. Vizetto-Duarte, H. Pereira, M.D. Laurenti, L. Campino, D. Levy, M. Uemi, L. Barreira, L. Custódio, L.F.D. Passero, J.H.G. Lago, J. Varela, Exp. Parasitol. 174, 1 (2017)

135. C. Girardi, N. Fabre, L. Paloque, A.P. Ramadani, F. Benoit-Vical, G. González-Aspajo, M. Haddad, E. Rengifo, V. Jullian, J. Ethnopharmacol. 170, 167 (2015)

136. B.B. Cota, L.G. Tunes, D.N.B. Maia, J.P. Ramos, D.M. De Oliveira, M. Kohlhoff, T.M.A. de Alves, E.M. Souza-Fagundes, F.F. Campos, C.L. Zani, Mem. Inst. Oswaldo Cruz 113, 102 (2018)

137. C.R. Novello, E. Düsman, R.B. Balbinot, J.C. de Paula, C.V. Nakamura, J.C.P. de Mello, M.H. Sarragiotto, Nat. Prod. Res. 0, $1(2020)$

138. C.B. Naman, A.D. Gromovsky, C.M. Vela, J.N. Fletcher, G. Gupta, S. Varikuti, X. Zhu, E.M. Zywot, H. Chai, K.A. Werbovetz, A.R. Satoskar, A.D. Kinghorn, J. Nat. Prod. 79, 598 (2016)

139. M.G.F. de Medeiros, A.C. da Silva, A.M.G.L. das Citó, A.R. Borges, S.G. de Lima, J.A.D. Lopes, R.C.B.Q. Figueiredo, Parasitol. Int. 60, 237 (2011)

140. V. Smyrniotopoulos, C. Merten, M. Kaiser, D. Tasdemir, Mar. Drugs 15, 1 (2017)

141. O. Chiboub, I. Sifaoui, J. Lorenzo-Morales, M. Abderrabba, M. Mejri, J.J. Fernández, J.E. Piñero, A.R. Díaz-Marrero, Mar. Drugs 17, 1 (2019).

142. D.C. Soares, M.M. Szlachta, V.L. Teixeira, A.R. Soares, E.M. Saraiva, Mar. Drugs 14, (2016).

143. A.R. Díaz-marrero, A. López-arencibia, C.J. Bethencout-estrella, F. Cen-pacheco, I. Sifaoui, A. Hernández, M.C. Duque-ramírez, I. Universitario, D.B.A. González, I. Ag, C. De Investigaciones, B. De Canarias, U. De La, L. Ull, Bioorg. Chem. 92, (2019)

144. C. Imperatore, R. Gimmelli, M. Persico, M. Casertano, A. Guidi, F. Saccoccia, G. Ruberti, P. Luciano, A. Aiello, S. Parapini, S. Avunduk, N. Basilico, C. Fattorusso, Mar. Drugs 18, (2020). 
145. T. Meza-Menchaca, A. Ramos-Ligonio, A. López-Monteon, A.V. Limón, L.A. Kaluzhskiy, T.V. Shkel, N.V. Strushkevich, L.F. Jiménez-García, L.T.A. Moreno, V. Gallegos-García, J. SuárezMedellín, Á. Trigos, Biomolecules 9, (2019).

146. S. Mukherjee, W. Xu, F.F. Hsu, J. Patel, J. Huang, K. Zhang, Mol. Microbiol. 111, 65 (2019)

147. L.P. Borba-Santos, G. Visbal, T. Gagini, A.M. Rodrigues, Z.P. De Camargo, L.M. Lopes-Bezerra, K. Ishida, W. De Souza, S. Rozental, Front. Microbiol. 7, 1 (2016)

148. B. Lomenick, H. Shi, J. Huang, C. Chen, Bioorganic Med. Chem. Lett. 25, 4976 (2015)

149. C. Park, D.O. Moon, C.H. Ryu, B.T. Choi, W.H. Lee, G.Y. Kim, Y.H. Choi, Acta Pharmacol. Sin. 29, 341 (2008)

150. L. Pan, C.M. Lezama-Davila, A.P. Isaac-Marquez, E.P. Calomeni, J.R. Fuchs, A.R. Satoskar, A.D. Kinghorn, Phytochemistry 82, 128 (2012)

151. H.T. Takahashi, E.A. Britta, R. Longhini, T. Ueda-Nakamura, J.C. Palazzo De Mello, C.V. Nakamura, Planta Med. 79, 330 (2013)

\section{Affiliations}

\section{Patrick O. Sakyi ${ }^{1,2} \cdot$ Richard K. Amewu $^{1} \cdot$ Robert N. O. A. Devine ${ }^{2} \cdot$ Emahi Ismaila $^{2} \cdot$ Whelton A. Miller $^{3,4,5}$. Samuel K. Kwofie ${ }^{6,7}$}

Patrick O. Sakyi

patrick.sakyi@uenr.edu.gh; opsakyi@st.ug.edu.gh

Richard K. Amewu

ramewu@ug.edu.gh

Robert N. O. A. Devine

robert.devine.stu@uenr.edu.gh

Emahi Ismaila

Ismaila.emahi@uenr.edu.gh

Whelton A. Miller

wmiller6@luc.edu

1 Department of Chemistry, School of Physical and Mathematical Sciences, College of Basic and Applied Sciences, University of Ghana, P. O. BOX LG 56, Legon, Accra, Ghana

2 Department of Chemical Sciences, School of Sciences, University of Energy and Natural Resources, Box 214, Sunyani, Ghana
152. V. Leliebre-Lara, L.M. Fidalgo, E.M. Pferschy-Wenzig, O. Kunert, C.N. Lima, R. Bauer, Molecules 21, 1045 (2016)

153. E.S. Elkhayat, S.R.M. Ibrahim, G.A. Mohamed, S.A. Ross, Nat. Prod. Res. 30, 814 (2016)

154. G.H. Braun, H.P. Ramos, A.C.B.B. Candido, R.C.N. Pedroso, K.A. Siqueira, M.A. Soares, G.M. Dias, L.G. Magalhães, S.R. Ambrósio, A.H. Januário, R.C.L.R. Pietro, Nat. Prod. Res. 1 (2019)

155. A.A.S. Silva, S.M. Morais, M.J.C. Falcão, I.G.P. Vieira, L.M. Ribeiro, S.M. Viana, M.J. Teixeira, F.S. Barreto, C.A. Carvalho, R.P.A. Cardoso, H.F. Andrade-Junior, Phytomedicine 21, 1419 (2014)

156. L. Keller, J.L. Siqueira-neto, J.M. Souza, K. Eribez, G.M. Lamonte, J.E. Smith, W.H. Gerwick, Molecules 25, 1604 (2020)
3 Department of Medicine, Loyola University Medical Center, Maywood, IL 60153, USA

4 Department of Molecular Pharmacology and Neuroscience, Loyola University Medical Center, Maywood, IL 60153, USA

5 Department of Chemical and Biomolecular Engineering, School of Engineering and Applied Science, University of Pennsylvania, Philadelphia, PA 19104, USA

6 Department of Biomedical Engineering, School of Engineering Sciences, College of Basic \& Applied Sciences, University of Ghana, PMB LG 77, Legon, Accra, Ghana

7 Department of Biochemistry, Cell and Molecular Biology, West African Centre for Cell Biology of Infectious Pathogens, College of Basic and Applied Sciences, University of Ghana, P.O. Box LG 54, Accra, Ghana 